NIST Technical Note 2141

\title{
Simulations of Flow Over an Axisymmetric Hill
}

Rikhi Bose

DongHun Yeo

This publication is available free of charge from: https://doi.org/10.6028/NIST.TN.2141

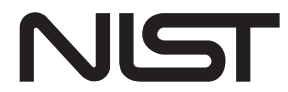

National Institute of Standards and Technology

U.S. Department of Commerce 
NIST Technical Note 2141

\title{
Simulations of Flow Over an Axisymmetric Hill
}

\author{
Rikhi Bose \\ DongHun Yeo \\ Materials and Structural Systems Division \\ Engineering Laboratory
}

This publication is available free of charge from: https://doi.org/10.6028/NIST.TN.2141

February 2021

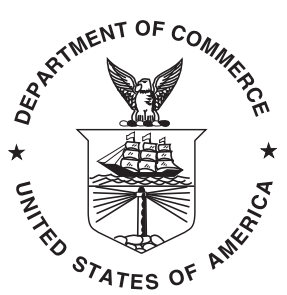

U.S. Department of Commerce Wynn Coggins, Acting Secretary

National Institute of Standards and Technology James K. Olthoff, Performing the Non-Exclusive Functions and Duties of the Under Secretary of Commerce for Standards and Technology \& Director, National Institute of Standards and Technology 
Certain commercial entities, equipment, or materials may be identified in this document in order to describe an experimental procedure or concept adequately. Such identification is not intended to imply recommendation or endorsement by the National Institute of Standards and Technology, nor is it intended to imply that the entities, materials, or equipment are necessarily the best available for the purpose.

National Institute of Standards and Technology Technical Note 2141 Natl. Inst. Stand. Technol. Tech. Note 2141, 47 pages (February 2021) CODEN: NTNOEF

This publication is available free of charge from: https://doi.org/10.6028/NIST.TN.2141 


\begin{abstract}
In this report, high-Reynolds number turbulent flow around an axisymmetric hill is studied using large-eddy and unsteady Reynolds-averaged Navier-Stokes simulations and the results are extensively compared against experiment. The boundary-layer thickness of the incoming flow is about half of the height of the hill. In the process, the dynamic Smagorinsky sub-grid scale eddy-viscosity model and a precursor forcing technique to match the experimental mean velocity profile is implemented in the computational toolbox for wind engineering, windOF which is based on the open source computational fluid dynamic solver OpenFOAM. The results show that the windOF is capable of producing reliable results with large-eddy simulation (LES) for this complex flow which includes complicated flow topography due to smooth-wall separation, lee-side separation, reattachment and recovery behind the hill, etc. However, Unsteady Reynolds-Averaged Navier-Stokes (URANS) predictions demonstrate significant discrepancies in several flow features, specifically in the lee-side separation and recovery that are characteristic of the Reynolds-Averaged Navier-Stokes (RANS) models which only consider much smaller scale turbulence compared to the mean flow distortion scales relevant to this flow.
\end{abstract}

\title{
Key words
}

Axisymmetric hill; Complex topography; large-eddy simulation (LES); Reynolds-Averaged Navier-Stokes Simulation (RANS); Turbulence. 


\section{Table of Contents}

1 Introduction $\quad 1$

2 Numerical experimental setup $\quad 4$

2.1 Large-eddy simulations $\quad 5$

2.2 LES model: dynamic Smagorinsky

$\begin{array}{lll}2.3 & \text { URANS } & 6\end{array}$

2.4 Flow solver 6

2.5 Computational grid 6

2.6 Boundary conditions 11

2.7 Calculations and Sampling 11

3 Results $\quad 13$

3.1 Precursor LES: Inflow generation 13

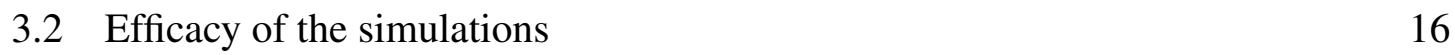

3.3 Results: Comparison with experiment 18

3.4 Upstream-side flow 31

4 Conclusion $\quad 34$

References $\quad 39$

\section{List of Figures}

Fig. 1 Schematic of the setup for the numerical experiment.

Fig. 2 Grid resolutions in viscous wall unit at the inlet of the main LES domain in the (a) wall-normal, $(b)$ streamwise and spanwise directions. Grid stretching ratio is also shown for grids in all three directions. The $y$ - and $z$ - grids remained same for both precursor and main simulations.

Fig. 3 Schematic views of the computational grid in the midspan $(z=0)$ plane: $(a)$ the full streamwise domain and $(b)$ zoomed-in view close to the hill. Every fourth grid location has been shown in both $(x)$ and $(y)$ directions.

Fig. 4 Grid resolution in local viscous wall units for the LES calculation: $(a)$ streamwise resolution $(\Delta x+) ;(b)$ wall-normal resolution at the centroid of the first wall-adjacent layer of cells $\left(\Delta y_{1 / 2}+\right)$; (c) spanwise resolution $(\Delta z+)$.

Fig. 5 Grid resolution in local viscous wall units for the URANS calculation: $(a)$ streamwise resolution $(\Delta x+) ;(b)$ wall-normal resolution at the centroid of the first wall-adjacent layer of cells $\left(\Delta y_{1 / 2}+\right) ;(c)$ spanwise resolution $(\Delta z+)$.

Fig. 6 Scaled wall-normal profiles of $(a)$ time-averaged streamwise velocity $(<u>$ $\left./ U_{0}\right)(b)$ turbulent kinetic energy and principal Reynolds stresses from the precursor simulation compared with the experimental results in [7].

Fig. 7 Frequency spectra of the fluctuating velocity components at three wall-normal heights $y+\sim 15,150$ and 1500 calculated in wall unit. 
Fig. 8 Spanwise autocorrelation of the fluctuating streamwise velocity at three wallnormal heights $y+\sim 15,150$ and 1500 calculated in wall unit.

Fig. 9 Ratio of the mean SGS eddy viscosity and molecular viscosity $\left.\left(<v_{S G S}\right\rangle / v\right)$ in the midspan $(z=0)$ plane in the main simulation domain.

Fig. 10 Ratio of $(a)$ the total eddy viscosity and molecular viscosity $\left(v_{T} / v\right)$ and $(b)$ the mean SGS eddy viscosity and the total eddy viscosity $\left(\left\langle v_{S G S}\right\rangle / v_{T}\right)$ in the midspan $(z=0)$ plane in the main simulation domain.

Fig. 11 Ratio of the mean turbulent eddy viscosity and molecular viscosity $\left(v_{T} / v\right)$ in the midspan $(z=0)$ plane from the URANS simulation.

Fig. 12 Contours of the pressure coefficient $\left(C_{p}\right)$ plotted in the horizontal $(x, z)$-plane as in experiment [6] (top frame) and from current LES (bottom frame). The circle in the bottom frame shows the outer circumference of the geometry of the hill.

Fig. 13 The pressure coefficient $\left(C_{p}\right)$ along the $x$-axis in the midspan $(z=0)$ plane compared with experimental data [6] and another high-resolution LES (the $L L$ simulation in [14]) and URANS [19].

Fig. 14 Streamlines based on the mean velocity field from the first layer of cells above the wall projected on to the bottom surface depicting the flow topology from LES (top frame) and URANS (bottom frame). The filled red (blue) circles indicate nodes (saddles).

Fig. 15 Streak-lines based on the mean velocity field depicting the flow topology at different wall-parallel planes at selected heights w.r.t. the bottom wall as in experiment [6] (four frames on the top) and from current LES (four frames on the bottom). The wall-normal heights of the selected planes have been shown in wall unit at inflow of the main simulation domain.

Fig. 16 Contour of the mean streamwise velocity $\langle u\rangle=0$ in the midspan $(z=$ 0) plane as in experiment [6] (top frame) and from current LES (middle frame) and URANS (bottom frame). Inplane velocity vectors are shown for URANS simulation in the bottom frame.

Fig. 17 Contours of the turbulent kinetic energy scaled by the reference velocity $\left(k / U_{0}^{2}\right)$ plotted in the midspan $(z=0)$ plane as in experiment [6] (top frame), from current LES (middle frame) and from the URANS simulation (bottom frame).

Fig. 18 Contours of mean streamwise vorticity $\left(\left\langle\omega_{x}\right\rangle\right.$ in $\left.H z\right)$ plotted in wallnormal $(y, z)$-planes at chosen streamwise locations from the main LES. From $(a)$ to $(f)$, the streamwise locations are $x / H=0,0.5,1,1.5,2$ and 2.5 , respectively. Vorticity patches based on their origin upstream of the location $x / H=1.5$ are marked in frame $(d)$. See the main text for detail.

Fig. 19 Wallnormal profiles of the scaled time-mean $(a)-(e)$ streamwise $\left(<u>/ U_{0}\right)$ and $(f)-(j)$ spanwise $\left(\langle w\rangle / U_{0}\right)$ velocities at $x / H=3.63$ from LES (solid lines) and URANS (dashed lines) compared with experiment (symbols). From top to bottom the spanwise locations are $z / H=0,0.32,0.65,1.1,1.79$. 
Fig. 20 Wallnormal profiles of the scaled $(a)-(e)$ turbulent kinetic energy $\left(k / U_{0}^{2}\right)$ and $(f)-(j)$ one of the cross components of the Reynolds shear stresses $(<$ $u v>/ U_{0}^{2}$ ) at $x / H=3.63$ from LES (solid lines) and URANS (dashed lines) compared with experiment (symbols). From top to bottom the spanwise locations are $z / H=0,0.32,0.65,1.1,1.79$.

Fig. 21 Frequency spectra of the $(a)$ streamwise and $(b)$ spanwise velocity fluctuations at $x / H=3.63, y / H=0.358$ in the midspan $(z=0)$ plane compared with experiment (symbols). The dashed line corresponds to $-5 / 3-$ law.

Fig. 22 Profiles of the scaled time-mean $(a)-(d)$ streamwise $\left(<u>/ U_{0}\right)$ and $(e)-$ (h) spanwise $\left(<w>/ U_{0}\right)$ velocities at $x / H=-2$ in the $y$-direction from current URANS (dashed line) compared with the current LES (dotted line). From top to bottom the spanwise locations are $z / H=0,1,2,3$.

Fig. 23 Profiles of the scaled time-mean $(a)-(d)$ streamwise $\left(<u>/ U_{0}\right)$ and $(e)-$ (h) spanwise $\left(<w>/ U_{0}\right)$ velocities at $x / H=-1$ in the $y$-direction from current URANS (dashed line) compared with the current LES (dotted line). From top to bottom the spanwise locations are $z / H=0,1,2,3$.

Fig. 24 Profiles of the scaled time-mean $(a)-(d)$ streamwise $\left(<u>/ U_{0}\right)$ and $(e)-(h)$ spanwise $\left(\langle w\rangle / U_{0}\right)$ velocities at $x / H=0$ in the $y$-direction from current URANS (dashed line) compared with the current LES (dotted line). From top to bottom the spanwise locations are $z / H=0,1,2,3$.

Fig. 25 Topographic Speed-up Factor (TSF) at the crest of the hill plotted as a function of wall-normal distance $(y / H)$ from current URANS (dashed line) compared with the current LES (solid line). 


\section{Introduction}

Application of Computational Fluid Dynamics (CFD) in wind engineering applications is a challenging field which is emerging with the advancements in computational capability of modern super computers. To this end, a computational toolbox, windOF, is being developed in the Structures Group of the Engineering Laboratory at the National Institute of Standards and Technology based on the open source CFD software, OpenFOAM (Open Field Operation And Manipulation) [1]. Recent endeavor in the group is to use the windOF toolbox to study atmospheric boundary-layer (ABL) flow problems $[2,3]$. Wind engineering problems often deal with high-Reynolds number turbulent flow over complex topography. Flow over a three-dimensional hill is a canonical problem of that class which possesses all the complexities encountered in flows over complex topographies, such as, reorientation of boundary-layer vorticity, flow acceleration and deceleration, unsteady separation and reattachment, separated shear layer and its interaction with upstream boundary-layer vorticity and so on. In this work, flow over a smooth three-dimensional axisymmetric hill is investigated using the windOF software. The chosen flow is popular for validating turbulence models and their efficacy in capturing flow dynamics of three-dimensional separation from curved surfaces as detailed data is available from several wind-tunnel experiments performed for this flow.

In an experimental campaign over a few years, the aforementioned flow around the smooth-wall axisymmetric hill geometry was studied (see [4-7]). These experimentalists used high-precision state of the art techniques such as, Laser-Doppler Velocimetry (LDV) and Hot-Wire Anemometry (HWA) in obtaining a very detailed perspective of the flow. Despite the flow Reynolds number being high (w.r.t. the hill height $R e_{H}=\frac{U_{0} H}{v}=130,000$ ), it is within the realms of high fidelity simulations, such as large-eddy simulation (LES), detached eddy simulation (DES) etc. on modern super computers. Since then several numerical efforts, such as, the works by [8-15] to name a few, have investigated this flow. A great deal of detail has emerged making this flow an obvious choice for validation of the windOF. This report provides a detailed description of the current effort that explores this flow with windOF including extensive validation of results with the experimental data.

Many features of this flow are counterintuitive. It is normally assumed that high-fidelity simulations of turbulent flows is able to replicate experimentally obtained pressure distribution relatively easily. For this flow on the contrary, the pressure distribution is extremely sensitive to several factors influencing the simulation, e.g., the lee-side separation is very thin and extremely sensitive to grid resolution in all directions. Turbulence characteristics of the approach flow are also important. It is found in this work that the pressure field in the lee-side separation region is highly sensitive to the separation process. Several factors are involved. As in three-dimensional separation from smooth curved surfaces, instantaneous separation for this flow is intermittent and varies substantially over space and time. The vortices in the separated shear layer interact with the recirculation region. The outer flow also interacts with the separating flow. Additional interactions take place at the point of reattachment due to impinging shear layer and the consequent pressure fluctuations propa- 
gate upstream towards the recirculation zone. The pressure field on the other hand dictates the instantaneous separation location. Therefore, for predicting this flow with acceptable precision, precise representation of all influencing factors is important.

In this work, LES of this flow has been performed. So far, this framework is largely limited to specific in-house LES codes used by small research groups all over the world. OpenFOAM provides a common platform for researchers which has been utilized in recent past to advance the field of applied fluid dynamics of turbulent flows. The sub-grid scale (SGS) model used in this study is the SGS eddy-viscosity type dynamic Smagorinsky model $[16,17]$ which has been widely used for non-equilibrium turbulent flows. A variant of the dynamic Smagorinsky model proposed by Lilly [17] was implemented in windOF. The dynamic Smagorinsky model was earlier successfully used for this flow [14]. Additionally, to prescribe a realistic turbulent boundary layer at the inlet of the computational domain containing the hill geometry, a precursor simulation technique was developed in windOF that can emulate experimental mean velocity and turbulent kinetic energy. This forcing technique is also widely used in the turbulent flow simulation community for generating realistic inflow conditions (see [18]). In this methodology, a forcing term is added on the right-hand side of the momentum conservation equations at each time step that can vary both temporally and spatially. It has been shown in this report that the implemented forcing technique is able to replicate the mean velocity field obtained in experiments [7] and the instantaneous cross-stream planes generated using this method may be used as inflow boundary conditions to simulate realistic turbulent flows over complex topography, such as, the axisymmetric hill.

Due to the computational constraint imposed by these methods at high Reynolds number, high-fidelity simulations such as LES is a challenge for wind engineering applications. In particular, Reynolds averaged Navier Stokes (RANS) simulations have been more popular in industrial computational wind engineering for topographic investigation. RANS turbulence models model the whole turbulence spectrum compared to the LES in which the larger eddies are resolved by the computational grid. Therefore, the resolution requirements for RANS are feasible even for very high Reynolds number flows. For the separational flow studied herein, one might expect RANS to perform well. As both the separated shear layer and recirculation region are very thin, intuitively, it might appear that this flow may not be influenced by large scale dynamics. However, this flow is dominated by large scale dynamics such as the mean flow distortion scales [14]. Because RANS models are formulated and tested for flows with turbulence scales much smaller than the mean flow distortion scales, perhaps thats is why almost all efforts with RANS have been unsuccessful in predicting this flow. All the RANS calculations show similar discrepancies, such as, very large recirculation region, erroneous mean flow predicted in the separation and flow recovery regions, etc (see [8-10, 19]). To gauge windOF's performance with RANS, this flow was also simulated with the widely used $k-\omega$ shear stress transport (SST) single-point two-equation RANS model [20]. The results from the unsteady RANS (URANS) simulation is compared with the LES and experiments. Of specific interest was the upstream-side flow where the flow accelerates over the hill. In wind engineering applications, topographic 
effect on wind speed is important for which RANS is widely employed. In this work, mean velocity profiles from URANS and LES are compared in the upstream side of the hill.

This manuscript is structured as follows. The numerical methodology relevant to LES and URANS are discussed in detail in Section 2. In Section 3, LES and URANS simulation results are presented from both the precursor simulation and the simulation with the hill geometry. The results are thoroughly validated against experiments in this section. Finally, conclusions are drawn in Section 4. 


\section{Numerical experimental setup}

The computational setup is for an axisymmetric hill geometry described by the following function for the height as a function of the radius $(r)$ from the symmetry axis,

$$
\frac{y(r)}{H}=-\frac{1}{6.04844}\left[J_{0}(\Lambda) I_{0}\left(\Lambda \frac{r}{2 H}\right)-I_{0}(\Lambda) J_{0}\left(\Lambda \frac{r}{2 H}\right)\right]
$$

where, $\mathrm{H}$ is the height of the hill, $\Lambda=3.1926, J_{0}$ is the Bessel function of the first kind and $I_{0}$ is the modified Bessel function of the second kind. The radius of the hill at its base is $2 \mathrm{H}$. Authors in [4] and [7] performed measurements of the flow around the model of a hill with height $H=0.078 \mathrm{~m}$ and this geometry is mounted on the floor of a $0.91 \mathrm{~m}$ wide, $0.25 \mathrm{~m}$ high and $7.62 \mathrm{~m}$ long test section of a low-speed wind tunnel. The maximum incoming velocity is $U_{0}=27.5 \mathrm{~ms}^{-1}$. Based on the hill height, the Reynolds number $\operatorname{Re}_{H}=\frac{U_{0} H}{v}=1.3 \times 10^{5}$, where, $v$ is the molecular viscosity. The experiments were performed in flow with freestream turbulence intensity $\sim 0.1 \%$. In the absence of the model, a turbulent boundary layer is obtained with thickness $\delta \sim H / 2$ at the location of the top of the hill ( $x=0$ in our coordinate setup). The momentum-thickness $(\theta)$ Reynolds number is $\operatorname{Re}_{\theta}=\frac{U_{0} \theta}{v} \sim 7300$.

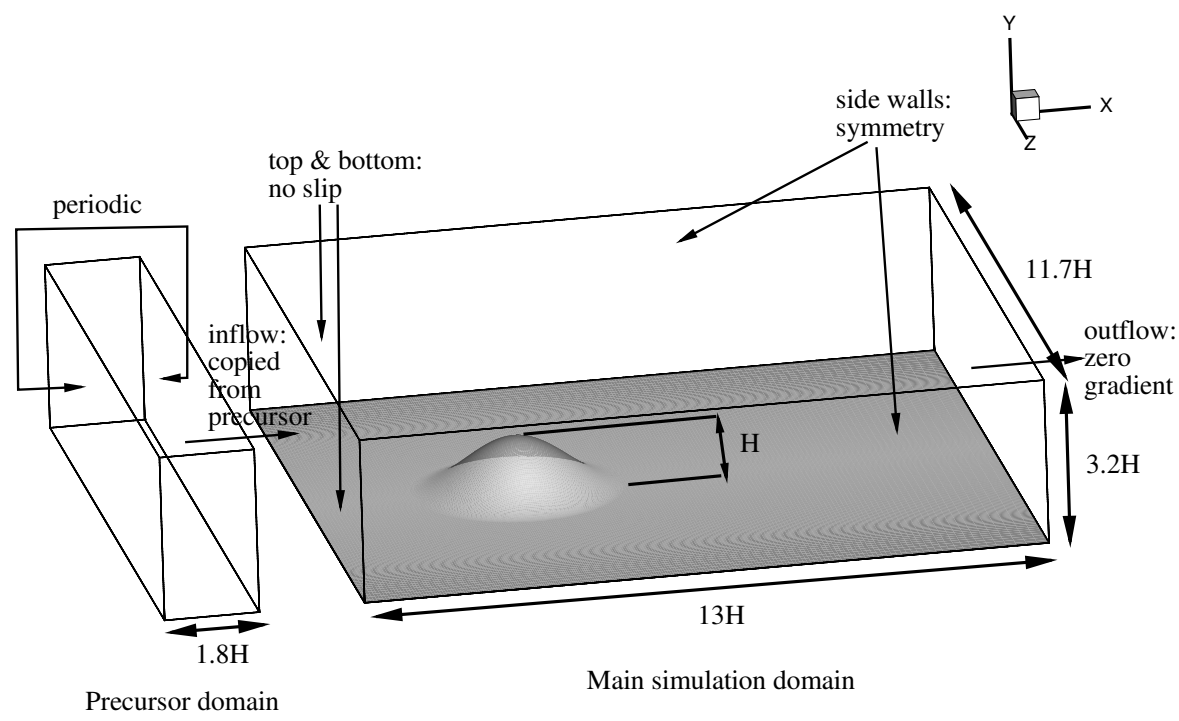

Fig. 1. Schematic of the setup for the numerical experiment.

This flow configuration is studied in this work by means of large-eddy simulations (LES). The numerical experimental setup is shown in Fig. 1. Two simulations were performed. The hill simulation was performed in a rectangular box stretching $13 \mathrm{H} \times 3.2 \mathrm{H} \times$ $11.7 \mathrm{H}$ in the streamwise $(x)$, wallnormal $(y)$ and spanwise $(z)$ directions, respectively. From here onwards, we refer to this as the main simulation. As in experiments, the hill surface 
is prescribed in the description of the bottom surface of the domain. For the purpose of prescribing unsteady turbulent inflow as in experiments, a precursor calculation was also performed in another rectangular box of length $1.8 \mathrm{H}$. The wall-normal and spanwise extents of the precursor simulation domain are same as the main simulation domain with the hill.

\subsection{Large-eddy simulations}

High-fidelity simulations are essential for capturing high-Reynolds number turbulent flow dynamics. However, for high-Reynolds number turbulent flows, the cost of direct numerical simulations (DNS) becomes exorbitant, if not out of scope of a modern supercomputer. Hence, LES is the viable option pursued for the present calculations. The incompressible continuity and momentum equations for the filtered velocity $\left(\tilde{u}_{i}\right)$ and pressure $(\tilde{p})$ fields are solved.

$$
\begin{gathered}
\partial_{i} \tilde{u_{i}}=0 \\
\partial_{t} \tilde{u}_{i}+\partial_{j}\left(\tilde{u_{i}} \tilde{u_{j}}\right)=-\partial_{i} \tilde{p}+\partial_{j}^{2} \tilde{u}_{i}+\partial_{j} \tilde{\tau_{i j}}
\end{gathered}
$$

Here, $\tilde{\tau_{i j}}=\tilde{u_{i}} \tilde{u_{j}}-\widetilde{u_{i} u_{j}}$ is the sub-grid scale (SGS) stress tensor which is modeled in an LES. This term appears in the momentum equation due to an implicit filtering operation (represented by ' $\sim$ ') on the total flow quantities. As the name suggests, this term accounts for the SGS flow dynamics and its interaction with the resolved flow field. The resolved flow field is the solution of the equations on the underlying grid. The third term on the RHS in the expression for $\tilde{\tau_{i j}}$ cannot be computed directly, and therefore, an SGS model is required. In this work we used the widely used formulation of an eddy-viscosity dynamic Smagorinsky model proposed in [17].

\subsection{LES model: dynamic Smagorinsky}

As the nomenclature suggests, in eddy viscosity SGS models, the deviatoric part of the SGS stress tensor is modeled as $\tilde{\tau_{i j}}-\frac{1}{3} \tilde{\tau_{k k}} \delta_{i j}=2 v_{S G S} \tilde{S_{i j}}$, where, $v_{S G S}$ is the SGS eddy viscosity and $\tilde{S_{i j}}$ is the resolved rate-of-strain tensor. In a typical CFD code, the isotropic SGS kinetic energy is absorbed in a modified expression for pressure. In the Smagorinsky model, $v_{S G S}$ is calculated as a function of the instantaneous magnitude of the strain-rate

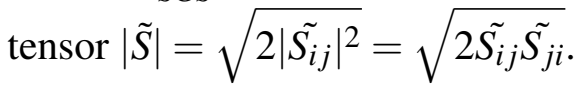

$$
v_{S G S}=C_{D} \Delta^{2}|\tilde{S}|
$$

Here, $\Delta$ is the filtering length scale which is proportional to the computational grid spacing. We calculated $\Delta=\left(\Delta V_{\text {cell }}\right)^{1 / 3}$ from the local cell volume $\left(\Delta V_{\text {cell }}\right)$. Unlike the Smagorinsky model, in the dynamic model $C_{D}$ is not a constant and computed at every time step. An explicit test filter of size $2 \Delta$ was used to calculate the subtest scale stresses. The 
difference between the resolved and subtest scale stresses is used to estimate the dissipation provided by the eddy viscosity in a dynamics model, which is proportional to $C_{D}$. In our simulations, calculation of $C_{D}$ is based on the method proposed by Lilly [17]. $C_{D}$ is smoothed by locally averaging it over the faces of a computational cell, and then used to compute the eddy viscosity using Eq. 4.

\subsection{URANS}

In practical topographic simulations, Reynolds-averaged Navier-Stokes (RANS) simulations have been preferred because of exorbitant computational costs involved for LES at high Reynolds numbers, frequently encountered in these applications. In RANS, as in Reynolds decomposition, the total flow quantities are decomposed into mean and turbulent fluctuating quantities. Unlike LES, in which only the SGS eddies are modeled, the whole spectrum of the turbulence quantities is modeled in RANS. For separational flows, the mean quantities are unsteady and vary with a large time scale. So, the time derivative terms are retained in unsteady RANS or, URANS. We investigate the efficacy of the widely used $k-\omega$ shear stress transport (SST) model, a two-equation eddy-viscosity model for this complex flow and compare its performance with the LES.

The implementation of the $k-\omega$ SST RANS model in OpenFOAM is based on a variant of the model proposed in [20]. As this model is very popular, there is a vast literature on its formulation and applications. So, for brevity, we will not repeat those details here.

\subsection{Flow solver}

Equations 2 and 3 were solved using the widely used open source CFD program OpenFOAM [1]. OpenFOAM solves the discretized equations in a collocated grid. The spatial discretization is performed with a finite volume method. Second order accurate Gauss linear interpolation for the fluxes from cell centers to cell faces was used for the calculation of the divergence and Laplacian terms of the equations integrated over a cell volume. The time integration is performed by the Crank-Nicolson scheme, hence, second order accurate. So, the overall accuracy of the solution is second order in both space and time. The OpenFOAM implementation of the PISO algorithm for unsteady incompressible flow, the 'pisoFoam' solver was used. In each PISO time step, momentum updation is performed once and the pressure correction twice. Two additional iterations of pressure correction to account for the grid nonorthogonality in each pressure correction step were used. The preconditioned conjugate gradient (PCG) and the preconditioned bi-conjugate gradient (PBiCGStab) algorithms were used for solving the pressure Poisson and momentum equations, respectively.

\subsection{Computational grid}

Fully resolved LES are performed, therefore, for both precursor and main simulations, fine grids have been used in all three directions. While the hill geometry is axisymmetric, the 
computational domain is Cartesian. So, for the main simulation, grid stretching was required in all directions. The number of grid points for the main simulation domain was $481 \times 165 \times 535$ for the computational domain $13 H \times 3.2 H \times 11.7 H$. For the precursor simulation, only the streamwise grid specification changes. 83 equally spaced grid points were used over a streamwise domain extent of $1.8 \mathrm{H}$. The total number of computational cells is $\sim 42$ million for the main simulation and $\sim 7.18$ million for the precursor simulation. The grids used for these well-resolved LES were generated based on gradual grid refinement from an earlier couple of simulations on increasingly coarser grids. This flow has been shown to be extremely sensitive to grid resolution $[11,13,14]$. Hence, a detailed description of the computational mesh is given.

For the LES, grid spacings in all three directions in viscous wall unit defined by the friction velocity at the inlet of the main simulation domain are shown in Fig. 2. The stretching ratio $(S R)$ of the grid spacings is also shown. In the left ordinate of Fig. 2(a), the scale is the wall-normal grid spacing in wall unit $\Delta y+$, while the right ordinate shows the grid's stretching ratio $(S R)$. In inlet-wall units, the height of the first cell adjacent to the wall corresponds to $\Delta y_{1}+\sim 0.8$. In the buffer layer, $\Delta y+\in[1.1,2.3] . \Delta y+\sim 6$ at $y+\sim 100$ in the logarithmic layer. $S R \sim 6.5 \%$ within wall-distance $y+\leq 50$ and decreases further away from the wall. The $y$-grid is only adequately refined for the lower wall. In the upper wall, the grid is refined; however, it is much coarser, and not shown herein.
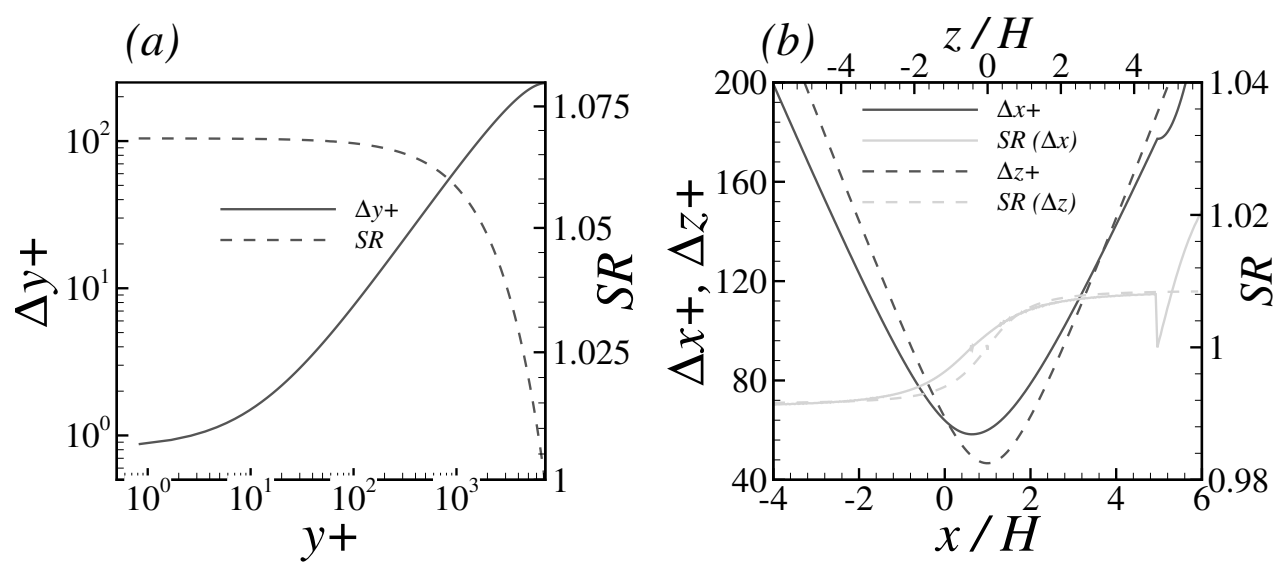

Fig. 2. Grid resolutions in viscous wall unit at the inlet of the main LES domain in the (a) wall-normal, $(b)$ streamwise and spanwise directions. Grid stretching ratio is also shown for grids in all three directions. The $y$ - and $z$ - grids remained same for both precursor and main simulations.

Due to the very high Reynolds number of the turbulent boundary layer considered, the streamwise and spanwise grid resolutions shown in Fig. 2(b) were relatively coarse compared to recommended well-resolved LES resolutions [21]. In Fig. 2(b), the lower and upper abscissae represent the domain extents in the $x$ and $z$ directions, respectively. The left ordinate again shows the grid spacings in wall unit, and the right ordinate shows the grid 
stretching ratio $(S R)$. This plot shows the target resolutions in the main simulation domain. The $x$-grid is gradually refined as the flow is expected to transition from an initial favorable pressure gradient (FPG) upstream of the hill to an adverse-pressure-gradient (APG) region in the lee-side where the separation takes place. In the lee side of the hill, post separation and reattachment, the flow is expected to recover to an equilibrium state. Thus, downstream of the expected separation point, the $x$-grid spacing is gradually increased. Beyond $x / H=$ 5 , grid stretching is increased as the flow in that regime is not the subject of the present work; hence, the resolution in that region may be deemed adequate. $z$-grid spacing is also gradually increased using the hyperbolic tangent function away from the center of the hill. With respect to the inlet-wall unit, $46 \leq \Delta z+\leq 91$ within the spanwise extent of the hill geometry. Minimum $\Delta x \approx$ minimum $\Delta z$ in the lee side of the hill in the midspan $(z=0)$ plane. $S R$ for both the $x$-grid and $z$-grid is within $1 \%$.

(a)

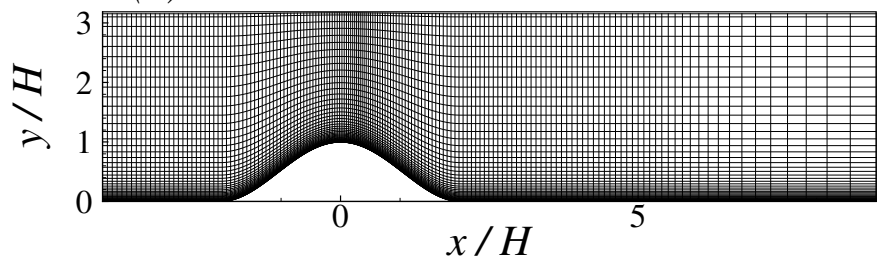

(b)

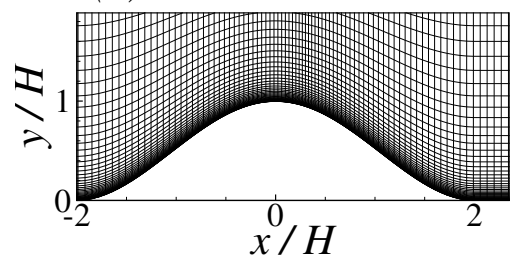

Fig. 3. Schematic views of the computational grid in the midspan $(z=0)$ plane: $(a)$ the full streamwise domain and $(b)$ zoomed-in view close to the hill. Every fourth grid location has been shown in both $(x)$ and $(y)$ directions.

Two views, one showing the whole extent of the streamwise computational domain, and another showing a zoomed-in view of the hill are shown in Fig. 3. These views demonstrate that the finest possible grid is used to resolve the APG and separation regions. The wallnormal grid resolution is significantly finer on the hill compared to the flat region.

The resolutions in all three directions of the main simulation grid in local wall units are plotted in Fig. 4. The dashed circle marks the circumference of outer edge of the hill. Although at inlet $\Delta x+\sim 175$, it gradually decreases downstream. $\Delta x+<100$ in most of the simulation domain except close to the inlet, the upstream region of the hill where flow accelerates, resulting in higher friction velocity and for $x / H>2.6$, where the grid spacing increases gradually. Maximum value of $\Delta x+\sim 150$ is obtained in the FPG region. Figure $4(b)$ shows the contours for $\Delta y+$ computed at the centroid of the first layer of cells adjacent to the wall. In the FPG region upstream of the hill, maximum $\Delta y_{1 / 2}+0.55$. So, the $y$-grid is adequate for resolving the near-wall turbulence. As mentioned earlier, the $z$-grid is stretched in that direction. Similar to the $x$-grid, peak value for $\Delta z+\sim 94$ is reached in the FPG region in the upstream side of the hill. Therefore, except considerably far away from the hill close to the side walls, $\Delta z+<50$ in most of the computational domain. Grid stretching was necessary to sufficiently resolve the region of the flow of significance. It must be noted, however, in OpenFOAM solver robustness is achieved by 

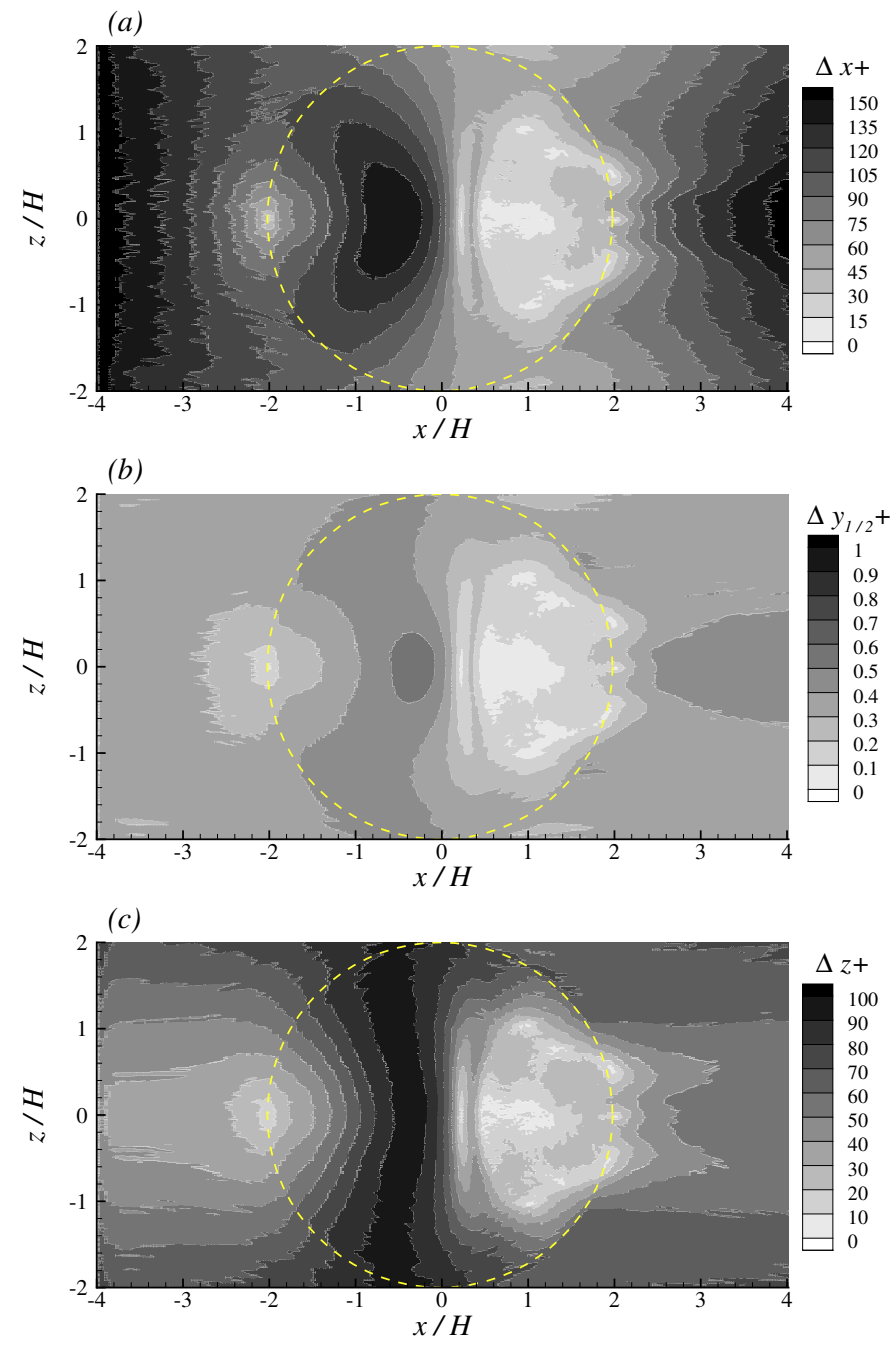

Fig. 4. Grid resolution in local viscous wall units for the LES calculation: (a) streamwise resolution $(\Delta x+) ;(b)$ wall-normal resolution at the centroid of the first wall-adjacent layer of cells $\left(\Delta y_{1 / 2}+\right) ;(c)$ spanwise resolution $(\Delta z+)$.

somewhat compromising the solution accuracy, which results in stringent grid requirements for OpenFOAM compared to other in-house solvers.

The computational domain used for the URANS calculation was same as the main LES simulation with the hill, i.e., $13 H \times 3.2 H \times 11.7 H$. The grid used for this flow is much coarser than the LES though. URANS simulations of this flow, although with a cubic eddy viscosity turbulence model was performed in [10]. Authors in [19] simulated this flow with the SST $k-\omega$ model. Our grid resolution was inspired by their grid resolutions. $111 \times 111 \times 131$ grid points were used in the $x, y$ and $z$-directions, which sums up to 1.57 million computational cells. As was for the main LES simulation, the grid is stretched 

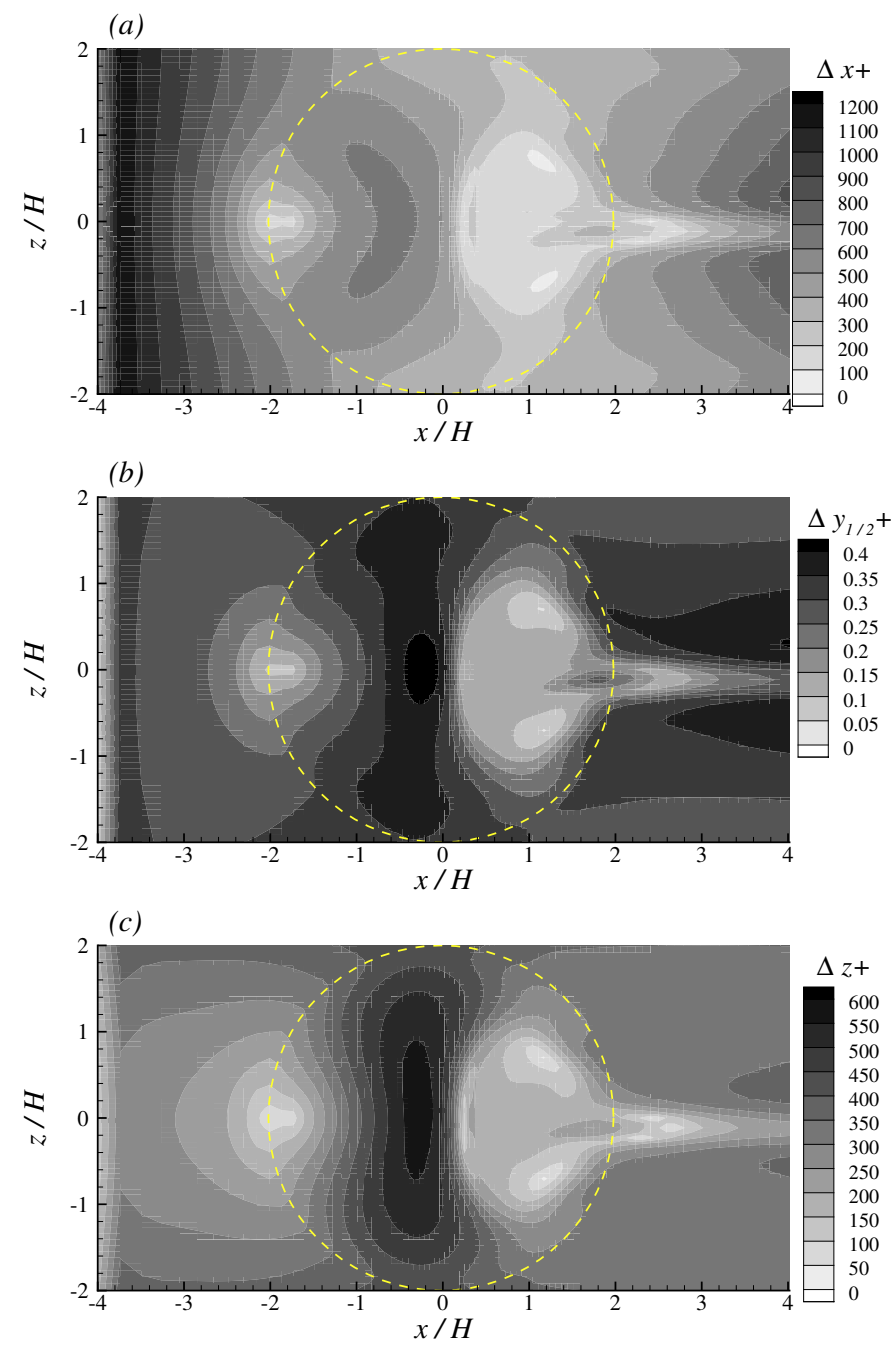

Fig. 5. Grid resolution in local viscous wall units for the URANS calculation: $(a)$ streamwise resolution $(\Delta x+) ;(b)$ wall-normal resolution at the centroid of the first wall-adjacent layer of cells $\left(\Delta y_{1 / 2}+\right) ;(c)$ spanwise resolution $(\Delta z+)$.

in all three directions. For the $k-\omega$ SST model, the wall-normal height of the first layer of cells, $\Delta y_{1}+<1$. With respect to the inlet wall units, $\Delta y_{1}+\sim 0.58$ in the flat region of the computational domain.

A figure similar to Fig. 4 is shown in Fig. 5 for the URANS calculation. As all the turbulence is modelled by the URANS, the streamwise and spanwise grid resolution in local viscous wall units is much coarser than the LES grid. Both the streamwise and spanwise grids for the URANS are at least five times coarser than the LES grid in the upstream side of the hill. The wallnormal resolution at the wall shown by the contour plot of $\Delta y_{1 / 2}+$ in Fig. $5(b)$ is similar to the LES resolution as a fine near-wall resolution is a necessity for the 
$k-\omega$ SST RANS model.

\subsection{Boundary conditions}

Boundary conditions used for the two LESs have been shown in Fig. 1. No slip and impermeabilitiy conditions have been used for velocity at the top and bottom walls. At the side walls, symmetry condition has been applied for velocity, so that the spanwise velocity component, $w=0$ there. At the outflow of the main simulation domain, a zero streamwise gradient condition was applied. At the inflow plane of the main simulation domain, unsteady turbulent velocity field from a computational plane normal to the flow direction saved at each time step of the precursor simulation was applied. Except inflow and outflow, boundary conditions at the four walls of the precursor simulation are same as the main simulation mentioned above. The precursor simulation is periodic in the streamwise direction for computing the fully developed turbulent boundary layer flow field. A zero-gradient condition was applied for pressure at all six boundaries of both the precursor and main simulation domains.

For the URANS simulations, the inlet boundary conditions for the velocity and turbulent kinetic energy $(k)$ fields are the steady profiles of the velocity and $k$ fields from the experiments (see Fig. 6). The inlet profile for the inverse time scale $\omega=\frac{\sqrt{k}}{C_{\mu} \kappa \Delta y}$ is obtained using the profile for $k$ and the wall-normal grid spacing $(\Delta y)$ at a given wall-normal height. In this expression, $\kappa$ is the constant from the log-layer equation and $C_{\mu}$ is the model constant. All other boundary conditions for velocity and pressure fields are same as the main LES simulation. $k=0$ is imposed at the no-slip top and bottom walls. At these walls,

$\omega=\frac{60 v}{0.075 \Delta y_{1 / 2}^{2}}$ as prescribed in the original formulations of Menter [20]. At the outflow the zero-streamwise gradient condition is imposed for $k$ and $\omega$. Similar to the LES, zerospanwise gradient boundary condition has been applied for these scalar quantities at the lateral walls.

\subsection{Calculations and Sampling}

While performing the LES calculations, the precursor simulation is carried out first. Once statistical stationarity is reached, calculation of statistics and saving data for prescribing at the inflow of the main simulation is initiated. For both LES and URANS simulations, time step in wall unit, $\Delta t+=\Delta t u *^{2} / v \approx 1.4$ w.r.t. the friction velocity $u *$ at inlet. As per recommendations in [22], although based on direct simulation of channel flow, time step used for the present calculations is just about small enough to temporally resolve turbulence adequately. The researchers in [22] noted that turbulence could not be sustained for $\Delta t+\geq$ 1.6 for low-Reynolds number channel flow for which a laminar solution was obtained. The very fine meshes impose severe requirement on computing time, and therefore, the chosen $\Delta t$ for the calculations made these feasible within the limits of our computational resources. Chosen $\Delta t$ resulted in a maximum Courant-Friedrichs-Lewy $(C F L)$ number [23] of $\sim 0.7$ for the URANS calculation. The maximum $C F L$ number at each timestep 
varied between $\sim 2.5$ and $\sim 8$ for the main LES. Although maximum $C F L$ for the main LES is quite high, it was intermittently obtained in a very few grid points near the maximum acceleration region and in the separated shear layer. The URANS and main LES were run initially to pass flow transients and for it to reach statistical stationarity when the flow averaging and data extraction were initiated. Then flow statistics were sampled for a period of $\Delta t_{a v}=176.28 \mathrm{H} / U_{0}$, which is sufficiently long to obtain smooth first and second order statistics. 


\section{Results}

Based on our experience of computing these LES in both fine grids as well as in preliminary coarser grids (results from which have not been presented herein), this flow is moderately sensitive to the boundary layer at the inflow of the main simulation. It has been argued previously, based on LES simulations on coarser grids with low-Reynolds number turbulent boundary layer flow used at inlet, that the effects of the incoming turbulence are weak on the flow separation [13]. However, there is no study showing that this is indeed the case for well-resolved LES. In fact, simulations of this flow with three types of velocity profiles (both laminar and turbulent) prescribed at inlet in [15] demonstrated some influence of the inflow condition on the downstream evolution of separation, reattachment and the vortical structures downstream of the hill, albeit at a lower Reynolds number. Therefore, in the following subsection, we first present results from the precursor simulation that demonstrate relevant attributes depicting flow structures of the incoming turbulence. These are followed by the results from the main LES and URANS simulations.

\subsection{Precursor LES: Inflow generation}

In the experiments $[4,7]$, precursor experiments were performed without the hill to measure boundary-layer profile and turbulence properties. Without the hill, a developed turbulent boundary layer with half hill-height thickness was obtained corresponding to $R e_{\theta} \sim 7300$. To prescribe a boundary layer matching the experimental profile at such a high Reynolds number at the inflow of the hill simulation domain, a forcing technique was necessary for the precursor simulation. This forcing technique for emulating experimental conditions in CFD simulations is widely accepted [18]. Unlike a fully developed turbulent flow simulation, in which, a constant body force term is added to the RHS of the u-component equation of eqn. 3 everywhere in the simulation domain at each time step maintaining either a constant pressure gradient or a constant mass flow rate, in this technique the added body force varies spatially and temporally. The following expresses the added body force at each time step varying across the cross-stream $(y, z)$ plane and also in time.

$$
f_{1}(y, z, t)=U_{e}(y)-<u(y, z, t)>_{x}
$$

Here, $U_{e}(y)$ is the mean streamwise velocity profile from the experiments, and $<$ $u(y, z, t)\rangle_{x}$ is the streamwise averaged instantaneous $u$-velocity. Hereafter, $\langle\zeta\rangle_{i}$ is the averaging of the variable $\zeta$ in the direction $i$. This expression preserves a constant streamwise mean flow rate at each time step. Moreover, due to it's variability in the cross-stream plane, it also maintains the desired profile of the mean velocity.

In Fig. 6, the temporally and spatially averaged mean streamwise velocity, $\frac{\left\langle\left\langle\langle u(y)\rangle_{x}\right\rangle_{z}\right\rangle_{t}}{U_{0}}=<$ $u>/ U_{0}$, turbulent kinetic energy $(k)$ and diagonal components of the Reynolds stresses are plotted against wall-normal distance in wall unit, $y+$. Exact match with experimental profile for the mean velocity is evident in Fig. 6(a). There is some discrepancy in the near-wall 

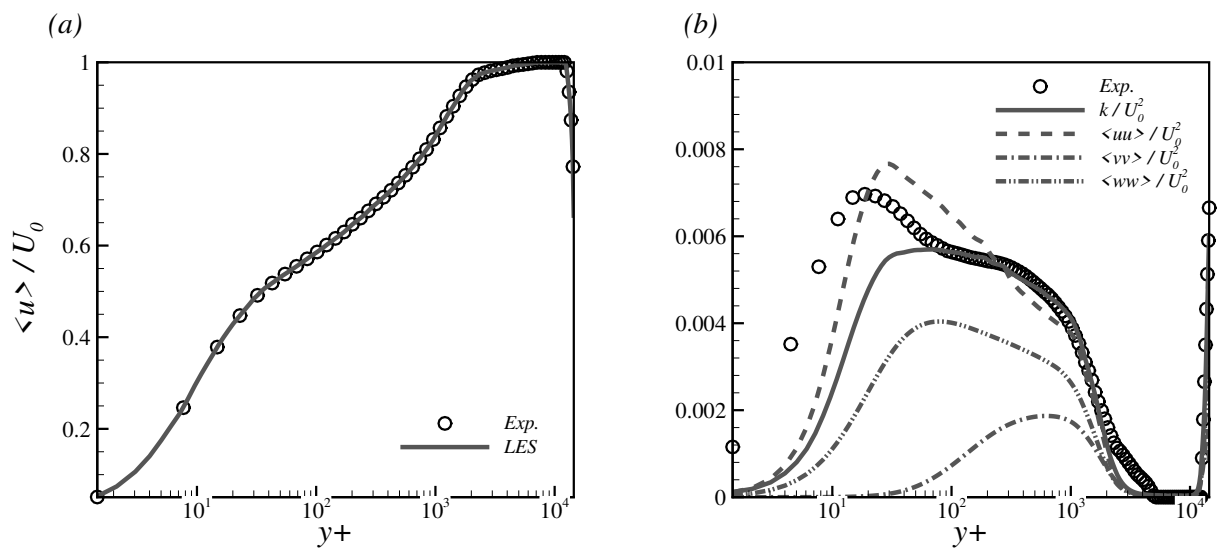

Fig. 6. Scaled wall-normal profiles of ( $a$ ) time-averaged streamwise velocity $\left(<u>/ U_{0}\right)(b)$ turbulent kinetic energy and principal Reynolds stresses from the precursor simulation compared with the experimental results in [7].

peak in $k$ (the peak in streamwise Reynolds stress is at $y+\sim 31$ instead of $y+\sim 15$ ), probably due to lower resolution in $x$ and $z$-directions compared to a standard LES requirement and also due to relatively large time step taken while integrating the solution in time. However, the near-wall peak values of the r.m.s. fluctuations scaled by $u *$ are 2.5, 1.23 and 1.8 for the $u, v$ and $w$ components which are in good agreement with the experimental observations by Counihan [24], who reported these as 2.5, 1.3 and 1.9, respectively.
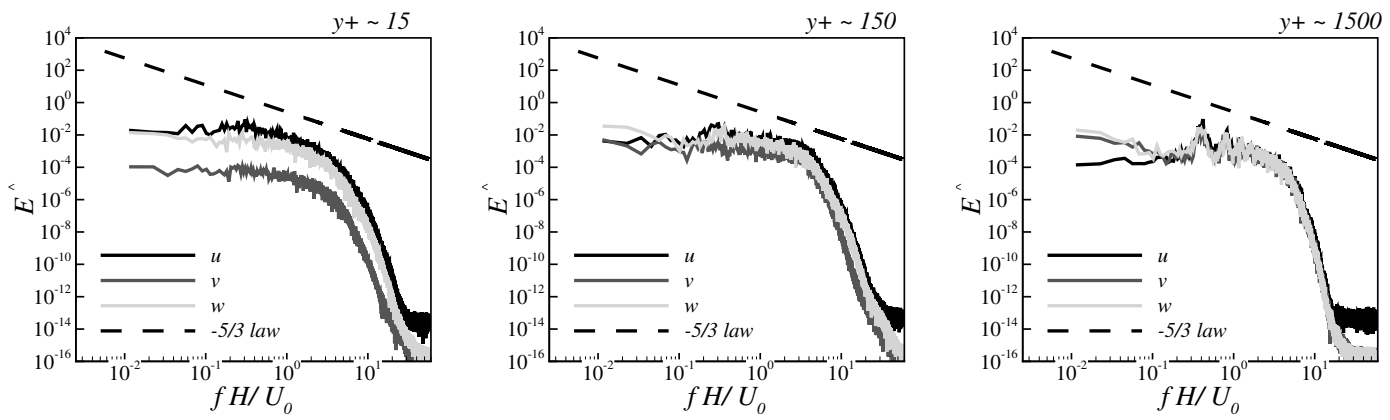

Fig. 7. Frequency spectra of the fluctuating velocity components at three wall-normal heights $y+\sim 15,150$ and 1500 calculated in wall unit.

Frequency spectra $(\hat{E})$ of the velocity fluctuations have been shown in Fig. 7 at three wall-normal heights, $y+\sim 15,150$ and 1500, which correspond to the buffer layer, logarithmic layer and outer region, respectively. Frequency ( $f$ in $H z$ ) is nondimensionalized by $H / U_{0}$. A clear $-5 / 3$-range emerges for over a decade in frequency for all three components. At $y+\sim 150$ and 1500, plots for three components collapse on each other indicating 
the isotropic nature of the small scale fluctuations. Because of the imposed streamwise periodicity, prominent peaks are obtained at $f H / U_{0} \sim 0.42$ and its higher harmonics, especially at $y+\sim 1500$ for all three components of velocity. Close to the wall, the effect of this periodicity on the main simulation results are however negligible as there is a considerable adjustment length in the main simulation domain before the flow approaches the hill. This was verified by plotting the frequency spectra at different streamwise stations close to the wall. However, further away from the wall, a peak corresponding to $f H / U_{0} \sim 0.42$ was also obtained in the frequency spectra calculated downstream of the hill far away from the wall in the main simulation domain (see Fig. 21).

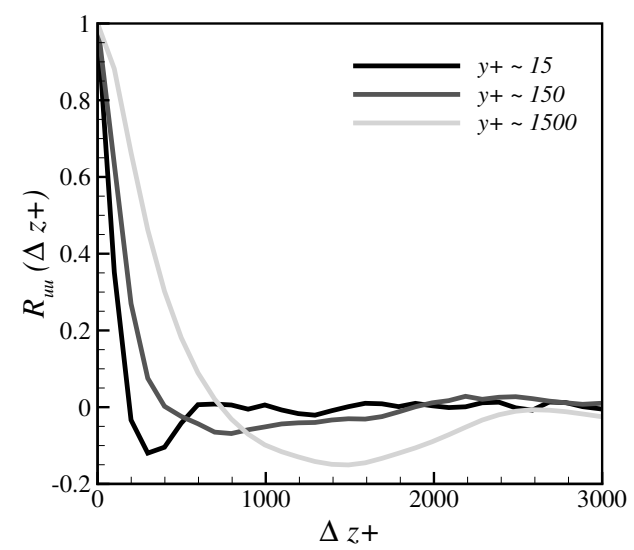

Fig. 8. Spanwise autocorrelation of the fluctuating streamwise velocity at three wall-normal heights $y+\sim 15,150$ and 1500 calculated in wall unit.

Two-point spanwise autocorrelation for the streamwise fluctuations $\left(u^{\prime}\right)$ expressed as $R_{u u}\left(\Delta z^{+}\right)=\frac{<u^{\prime}\left(z_{0}^{+}+\Delta z^{+}\right) u^{\prime}\left(z_{0}^{+}\right)>_{t}}{<u^{\prime}\left(z_{0}^{+}\right)^{2}>_{t}}$ has been plotted in Fig. 8 at the same wall-normal heights as in Fig. 7. The flow is not homogeneous in the spanwise direction. The grid is also stretched in that direction. Yet, for the precursor simulation, calculation of the spanwise autocorrelation function is justifiable within the purview of approximation. The velocity components were first interpolated to a grid with equidistant spacing. Close to the wall, vertical shear in the streamwise velocity induces streamwise elongated streaky flow structures. The size of those structures is reflected in Fig. 8. Close to the wall, the streaky structures are of size $\sim 600 \Delta z+$. Further away from the wall, the structures get bigger and wider. For very high Reynolds number turbulent shear flows, larger structures populate the region between the wall layer and the outer region. This plot shows that the near-wall structures are nominally resolved in the spanwise direction which results in the mismatch in the peak location of $k$ in Fig. 6. 


\subsection{Efficacy of the simulations}

The SGS model performance in a well-resolved LES is measured by computing the ratio of the mean SGS viscosity $\left\langle v_{S G S}\right\rangle=\left\langle v_{S G S}\right\rangle_{t}$ and the molecular viscosity $v$. It is assumed that the LES is accurate for $\left\langle v_{S G S}\right\rangle / v$ is in the order of 10 and below 100 [25]. The maximum of $\left\langle v_{S G S}\right\rangle / v \sim 8$ is obtained in the main simulation domain in the outer regions far away from the hill $(|z / H|>3.8)$ close to the under-resolved upper wall $(y / H>2.5)$ (because $v_{S G S} \propto \Delta^{2}$ in Eq. 4 and the grid spacing increases away from the hill in all three directions). In the lower wall region where the hill is mounted, $\left\langle v_{S G S}\right\rangle$ $/ v<2.5$ everywhere. In Fig. 9, this quantity is plotted in the midspan $(z=0)$ plane where the local maximum in the lower wall region is obtained in the main simulation domain. The maximum in this plane is found in the lee-side of the hill after separation and before reattachment. The peak value is obtained in the high shear region due to the separated shear layer away from the wall right after separation.

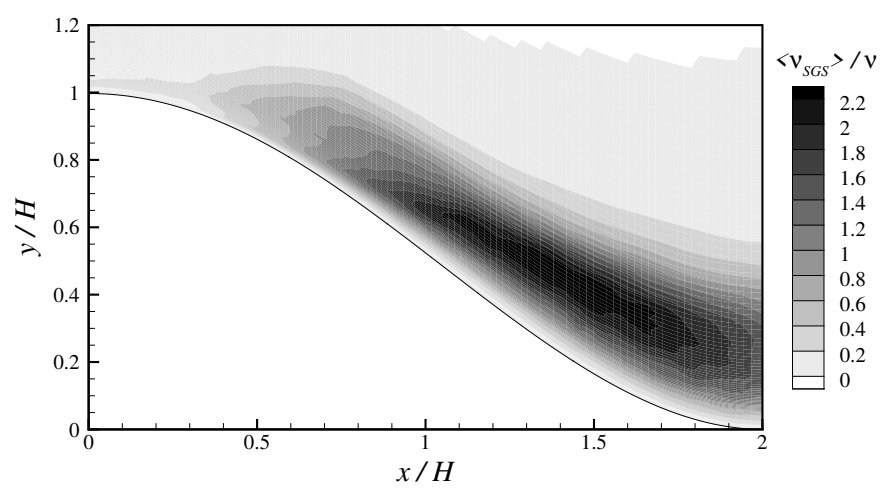

Fig. 9. Ratio of the mean SGS eddy viscosity and molecular viscosity $\left(<v_{S G S}>/ v\right)$ in the midspan $(z=0)$ plane in the main simulation domain.

A total eddy viscosity may be calculated based on the resolved Reynolds stresses from the LES. Linear eddy viscosity hypothesis relates the total eddy viscosity $\left(v_{T}\right)$ to the resolved Reynolds stresses as per following expression.

$$
2 v_{T}<S_{i j}>=-<u_{i}^{\prime} u_{j}^{\prime}>+\frac{2}{3} k \delta_{i j}
$$

Here, $<S_{i j}>$ is the mean rate of strain tensor. The second term on the RHS is added to make for satisfying the equality condition. From this expression, $v_{T}$ may be extracted as

$$
v_{T}=\frac{\left|-<u_{i}^{\prime} u_{j}^{\prime}>+\frac{2}{3} k \delta_{i j}\right|}{\left|2<S_{i j}>\right|}
$$

$v_{T}$ may be considered to be the total eddy viscosity provided by an URANS calculation for the same flow provided the same Reynolds stresses and mean rate of strain were 
obtained by the URANS calculation. Therefore, it is another performance parameter for an SGS model. The total eddy viscosity scaled by the molecular viscosity $\left(v_{T} / v\right)$ is plotted in Fig. 10 $(a)$ in the midspan plane. Expectedly, maximum value of $v_{T}$ is more than 1000 times larger than $v$. The maximum is obtained in the lee-side of the hill in the region where high values were obtained for $v_{S G S}$ in Fig. 9.
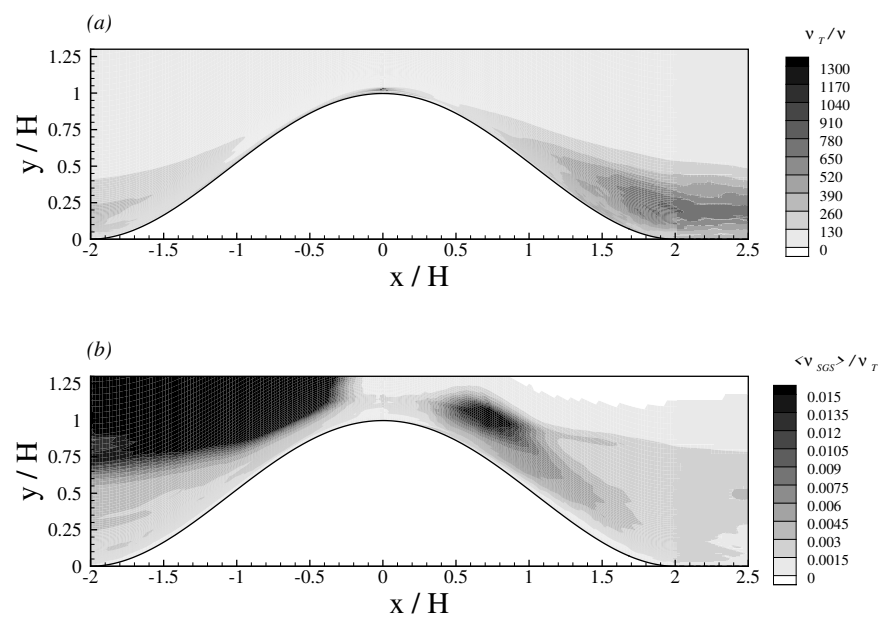

Fig. 10. Ratio of $(a)$ the total eddy viscosity and molecular viscosity $\left(v_{T} / v\right)$ and $(b)$ the mean SGS eddy viscosity and the total eddy viscosity $\left.\left(<v_{S G S}\right\rangle / v_{T}\right)$ in the midspan $(z=0)$ plane in the main simulation domain.

The ratio $<v_{S G S}>/ v_{T}$ quantifies the effect of the SGS model compared to the equivalent URANS model discussed above. This quantity is plotted in Fig. 10(b), again in the midspan plane. Maximum mean $v_{S G S}$ is only about $1.5 \%$ of the local $v_{T}$. High values were obtained either in the free stream far away from the wall where grid resolution is coarser or in a small patch in the lee-side around the region where the mean separation is initiated. This plot demonstrates that almost the whole of the resolved Reynolds stresses is due to the flow resolved by the grid and the SGS contribution is negligible towards the resolved stresses.

The ratio of the mean turbulent eddy viscosity and the molecular viscosity from the URANS simulation has been plotted in the midspan plane in Fig. 11. The contour levels in this plot are same as Fig. 10(a) for comparison. For the URANS simulation, this ratio is maximum $(\sim 2000)$ at the upper wall due to coarse grid used there. However, in the lee-side separation region, the peak value is similar to the maximum $v_{T} / v$ calculated from the LES. From the discussion of the results to be followed, it becomes clear that the streamwise local peak of this ratio is obtained at the junction of the recirculation region and the separated shear layer because of the high flow gradients there. This plot verifies that the LES was able to resolve the flow adequately. 


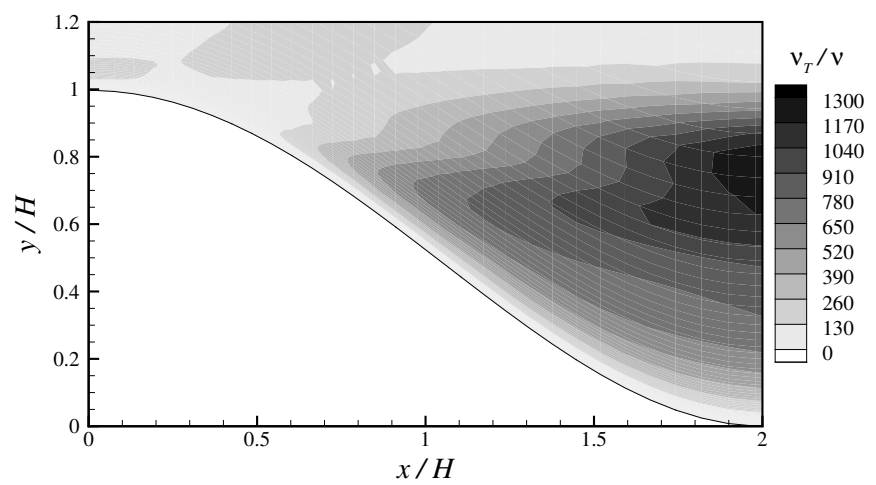

Fig. 11. Ratio of the mean turbulent eddy viscosity and molecular viscosity $\left(v_{T} / v\right)$ in the midspan $(z=0)$ plane from the URANS simulation.

\subsection{Results: Comparison with experiment}

The results from the main LES and URANS simulations have been extensively validated against experimental results. In the following discussion, any quantity $\langle f\rangle$ indicates time-averaged variable $f$.

In Fig. 12, contours of the pressure coefficient, $C_{p}=\frac{<p>-p_{0}}{\frac{1}{2} \rho U_{0}^{2}}$ have been plotted in the horizontal $(x, z)$-plane. The contour values have been projected from the bottom surface of the simulation to the horizontal plane. The top frame shows the experimental results and the bottom frame shows the results from current LES. In both plots, same contour values have been shown. Good agreement with the experiment is apparent. Downstream of the inlet plane, the flow decelerates first up to $x / H \sim-1.6$. Then onwards the flow accelerates over the slope of the hill. The minimum pressure is attained just upstream of the peak of the hill, at $x / H \sim-0.016$. The $C_{p}$ distribution in the initial deceleration and the following acceleration regions are indeed in very good agreement with experiment (except the minimum value of $C_{p}$; this is discussed later) all throughout the spanwise extent of the hill. The lee-side separation and reattachment is more complicated.

$C_{p}$ is also plotted in Fig. 13 along the midspan $(z=0)$ plane and compared with the experiment and another high-resolution LES [14] from available literature. The minimum in the present LES is lower than the value obtained in [14] which is in turn lower than the minima obtained in the experiment. However, the location of the minimum $C_{p}$ is the same as in the experiment. At the initiation of separation around $x / H \sim 0.22$, a very small hump is obtained in our simulation which is not seen in either of the other curves. Downstream of this location, the curves apparently collapse on each other until the plateau corresponding to the reattachment. The curves start flattening as the separation region thickens in the experiment for $x / H \geq 0.96$. Although similar trend is captured in the present LES, in the region $1 \leq x / H \leq 1.75$, the present curve overshoots the experiment. For this separational flow, $C_{p}$ is extremely sensitive to successful capturing of the separation 

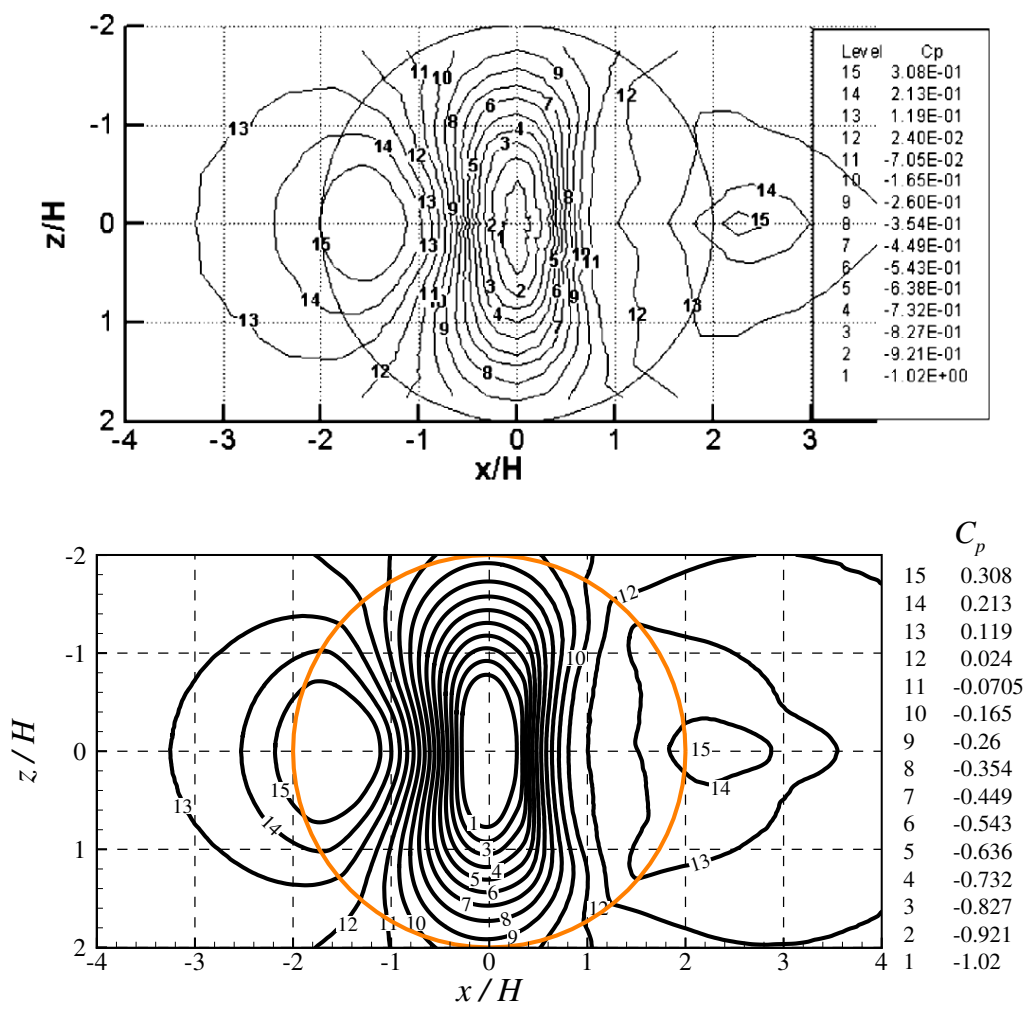

Fig. 12. Contours of the pressure coefficient $\left(C_{p}\right)$ plotted in the horizontal $(x, z)$-plane as in experiment [6] (top frame) and from current LES (bottom frame). The circle in the bottom frame shows the outer circumference of the geometry of the hill.

[12]. The overshoot in $C_{p}$ indicates a locally thinner recirculation layer close to the wall compared to the experiment.

$C_{p}$ distribution in the midspan plane for the URANS simulation has been also plotted and compared with the URANS simulation result from [19] in Fig. 13. Despite significant difference between URANS and LES simulation/experiment, excellent agreement between two URANS results is evident. The URANS predicts significantly thicker recirculation zone. This is reflected in the long flat portion in the $C_{p}$ distribution for $x / H>0.4$. Predicted separation, recirculation and reattachment characteristics are discussed in more detail in the following subsection.

In Fig. 14, streamlines of the skin-friction coefficient obtained from the mean flow projected on the horizontal $(x, z)$-plane have been shown. Streamlines from the LES are shown in the top frame while the URANS result has been shown in the bottom frame. This plot depicts the near-wall flow topology in one-half of the map around the hill about the symmetry plane. In the LES, the separation is triggered at $x / H \sim 0.22$ close to the midspan plane, for $0 \leq|z / H| \leq 0.5$. Post separation, the recirculation layer is very thin [4]. Close to $x / H \sim 0.4$, a reattachment zone can be observed in the midspan plane, followed 


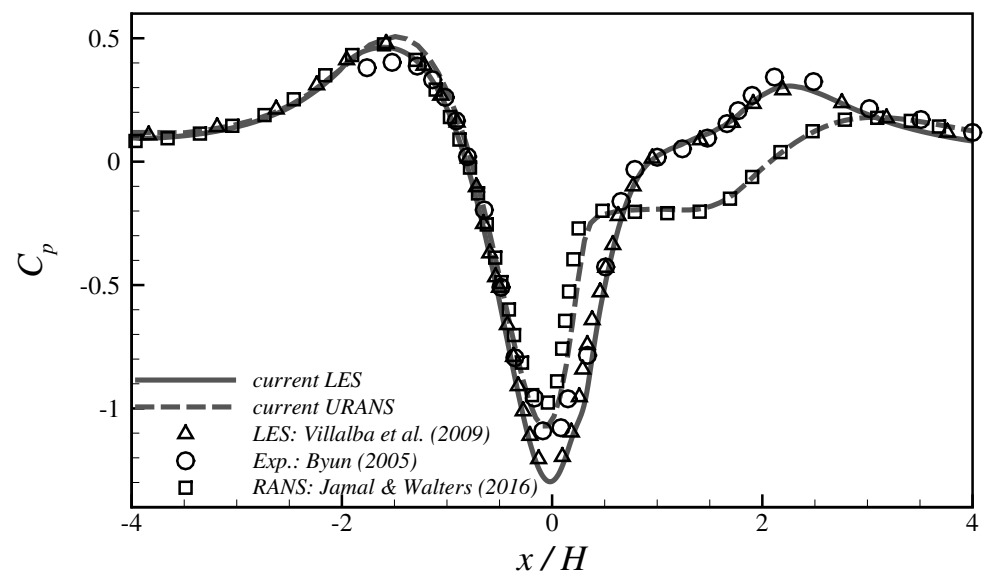

Fig. 13. The pressure coefficient $\left(C_{p}\right)$ along the $x$-axis in the midspan $(z=0)$ plane compared with experimental data [6] and another high-resolution LES (the $L L$ simulation in [14]) and URANS [19].

by another recirculation zone. In their first paper on the experiment, researchers in [4] reported a very complex flow pattern. From their oil-flow visualization, an initial separation was reported in the region $0.18 \leq x / H \leq 0.4$, and a second larger separation region was observed $0.4 \leq x / H \leq 2$ accompanied by adverse pressure gradient. $L D V$ measurements were used in the later experiment reported in [7]. They observed a separation triggered much later at $x / H=0.96$. The current LES results support the complex vortex structures observed in the former.

In the whole near-wall topology, five nodes are obtained for the LES marked by the filled red circles (streamlines either converge into or diverge away from these); the separation points along the midspan plane, the reattachment node at $x / H \sim 2$, and the two focal nodes in either side of the midspan plane. Accordingly, there are five saddle points (streaklines both converge towards and diverge away from these topological points). These are marked by the filled blue circles. These are, the saddle point at $x / H \sim 0.4$ and a pair of saddle points in either side of the symmetry plane upstream and downstream of the focal node. In an incompressible flow, the sum of the number of nodes must be equal to the number of saddle points [26]. Our results are different from the four nodes and four saddle points reported from the $G V R$ simulation in [14]. However, presence of the two focal points is consistent with both the LES simulations reported in [14] and experimental results reported in [7], but contradicts the observations presented in [4], who reported three pairs of vortical structures close to the wall. The reattachment node is consistent with both the experimental observations. The location of the focus and streak-line patterns around it is consistent with the $L L$ simulation results reported in [14]. Presence of the focal nodes indicate ejection of vortical structures from close to the wall. 

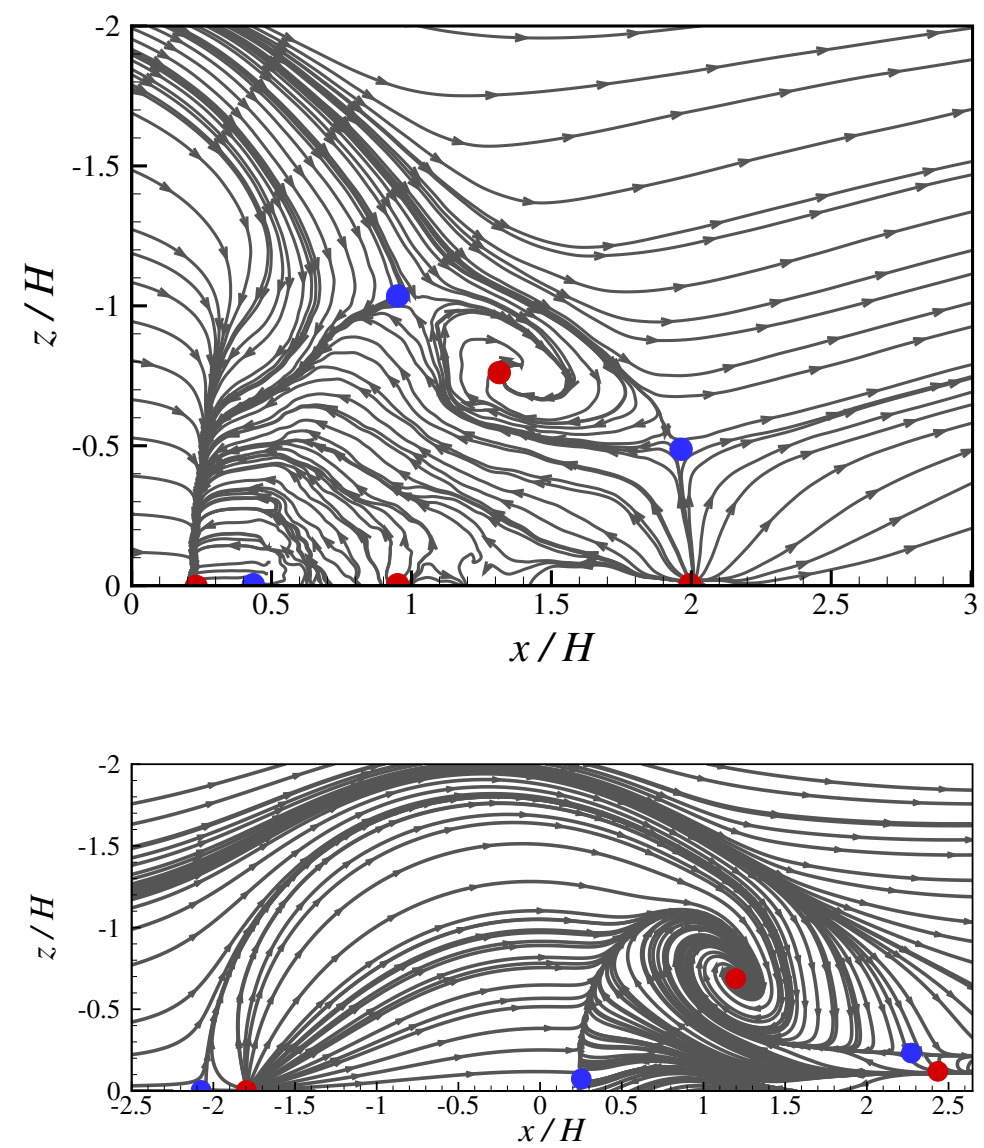

Fig. 14. Streamlines based on the mean velocity field from the first layer of cells above the wall projected on to the bottom surface depicting the flow topology from LES (top frame) and URANS (bottom frame). The filled red (blue) circles indicate nodes (saddles).

Near-wall flow topology from the URANS simulation has been plotted for the mean flow field in the bottom frame of Fig. 14. Four saddles (indicated by blue filled circles) and four nodes (red circles) are obtained for the URANS solution. Unlike the LES, a saddle and another node are obtained in the upstream side of the hill. Also, contrary to the LES, a saddle point is obtained at the lee-side separation location. The reattachment location denoted by the reattachment node along the midspan plane is further downstream of the reattachment node obtained in the experiment. Also, the reattachment node is accompanied by two saddle points, one in each side of the symmetry plane. The location of the focus is in good agreement with the experiment.

Farther away from the wall, flow topology for the LES simulation has been shown by plotting the streak-lines of the mean velocity field in Fig. 15 in wall-parallel planes at different heights above the bottom wall. Scaled turbulent kinetic energy contours $\left(k / U_{0}^{2}\right.$, 


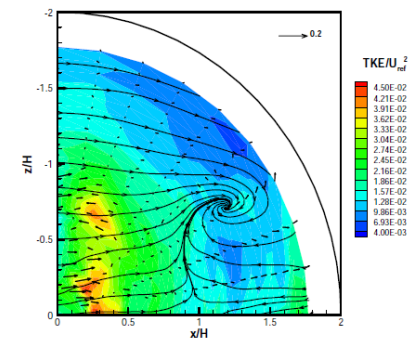

(a) $\mathrm{y}_{\mathrm{L} 0}{ }^{+}=11$

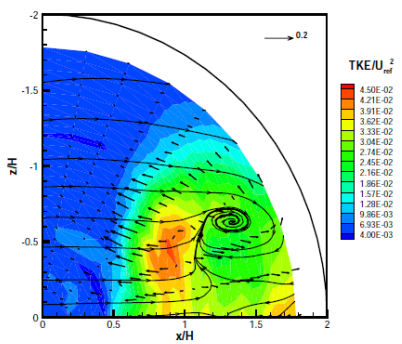

(c) $\mathrm{y}_{\mathrm{L} 0}{ }^{+}=200$

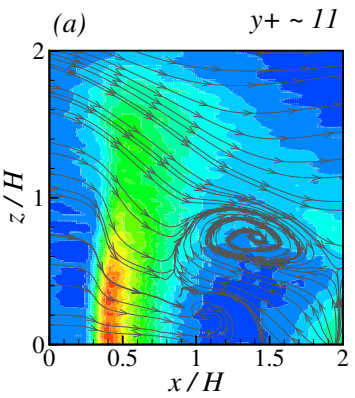

(c)

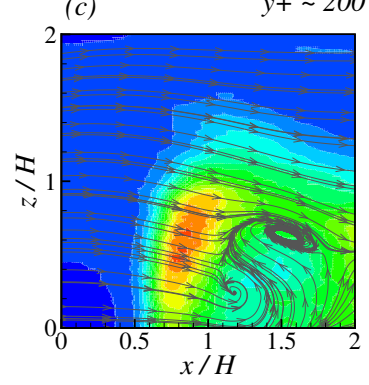

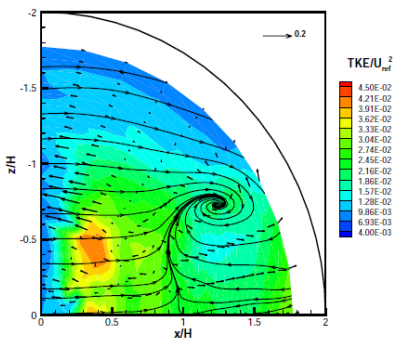

(b) $\mathrm{y}_{\mathbf{L} 0}{ }^{+}=41$

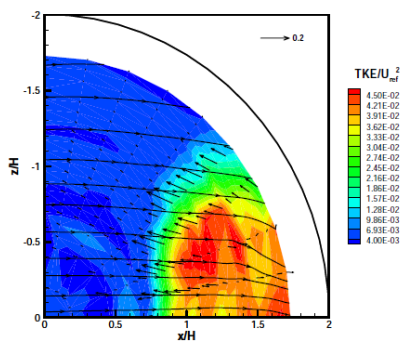

(d) $\mathrm{y}_{\mathbf{L} 0}{ }^{+}=\mathbf{4 5 6}$

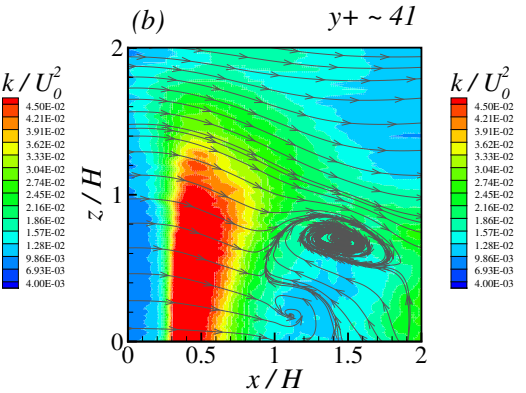

(d) $\quad y+\sim 456$

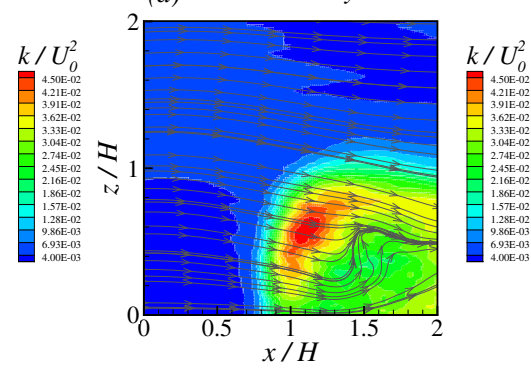

Fig. 15. Streak-lines based on the mean velocity field depicting the flow topology at different wall-parallel planes at selected heights w.r.t. the bottom wall as in experiment [6] (four frames on the top) and from current LES (four frames on the bottom). The wall-normal heights of the selected planes have been shown in wall unit at inflow of the main simulation domain. 
which is here onward denoted as $T K E$ ) are also shown in all these plots. Distance of these planes from the bottom wall are indicated w.r.t. the viscous wall-unit at the inlet of the main simulation domain. Four frames in the top shows the experimental results, and the bottom four are from the current LES. Close to the wall, at $y+\sim 11$, mean streak-lines initially curve inward towards the symmetry plane. The separation line along $x / H \sim 0.22$ observed in the top frame of Fig. 14 is not seen in this plot indicating that the first recirculation zone is very thin. In the experiment, flow separation only starts at $x / H \sim 0.96$ at this wall-normal height, followed by the streak-lines curving into the focus. In the current LES, close to the midspan plane, separation is initiated further downstream at $x / H \sim 1$.4. Farther away from the centerline, separation takes place at the same location as in the experiment. The location of the focus in the current LES corroborates the experiment. Good agreement is visually evident for the $T K E$ contours.

Similar trends for the streak-lines are seen further away from the wall at $y+\sim 40$. The location of the focus at all wall-normal heights from the LES are in good agreement with the experiment. But the initiation of separation is different for the LES where it is triggered further downstream. In fact, at $y+\sim 200$, in the range $1.5 \leq x / H \leq 2$, flow does not separate close to the midspan plane while clear separation was seen in the experiment at this wall-normal height. This indicates again as in the plot for $C_{p}$ in Figs. 12 and 13, the recirculation region is thinner in the LES compared to the experiment. The TKE contours, on the other hand, are in accordance with the experimental result. The flow topology demonstrates the complexity of the flow topology of this separational flow. The flow patterns vary significantly in both streamwise and spanwise directions in each plot. Also, significant alterations are evident from plot to plot as the chosen wall-normal height of the plane increases. These plots depict the complex three-dimensional pattern of separation and reattachment of the recirculation zones.

In the experiment, time-mean recirculation region in the midspan plane was reported by showing the zero-mean streamwise velocity $(\langle u\rangle=0)$ contour. This figure from [7] has been shown in the top frame of Fig. 16. In the middle and bottom frames of the figure, the same plot is shown from the current LES and URANS, respectively. For a threedimensional separation, $\langle u\rangle=0$ contour line may be considered a reasonable approximation of the mean recirculation region. For clarity, the in-plane velocity vectors are not shown in the bottom frame. In the LES, separation in this plane is initiated at the separation node shown in Fig. 14 at $x / H \sim 0.22$. Evidently, the recirculation region right after initiation of separation is very thin in the LES. In the experiment, separation starts further downstream at $x / H=0.96$. This discrepancy will be discussed later in more detail. The final reattachment is at $x / H \sim 2$ in the current LES, which is consistent with the experiment. However, the recirculation region is much thinner for the LES. This fact was reflected in the $C_{p}$ distribution in the midspan plane in Fig. 13. Also, the recirculation region is not continuous and three distinct recirculation zones are visible in the bottom frame. From our experience of simulating this flow, correctly capturing the separation and recirculation region is challenging as this is very sensitive to several factors. It is extremely sensitive to the grid resolution in the lee-side of the hill, the turbulent boundary condition at the inlet, 

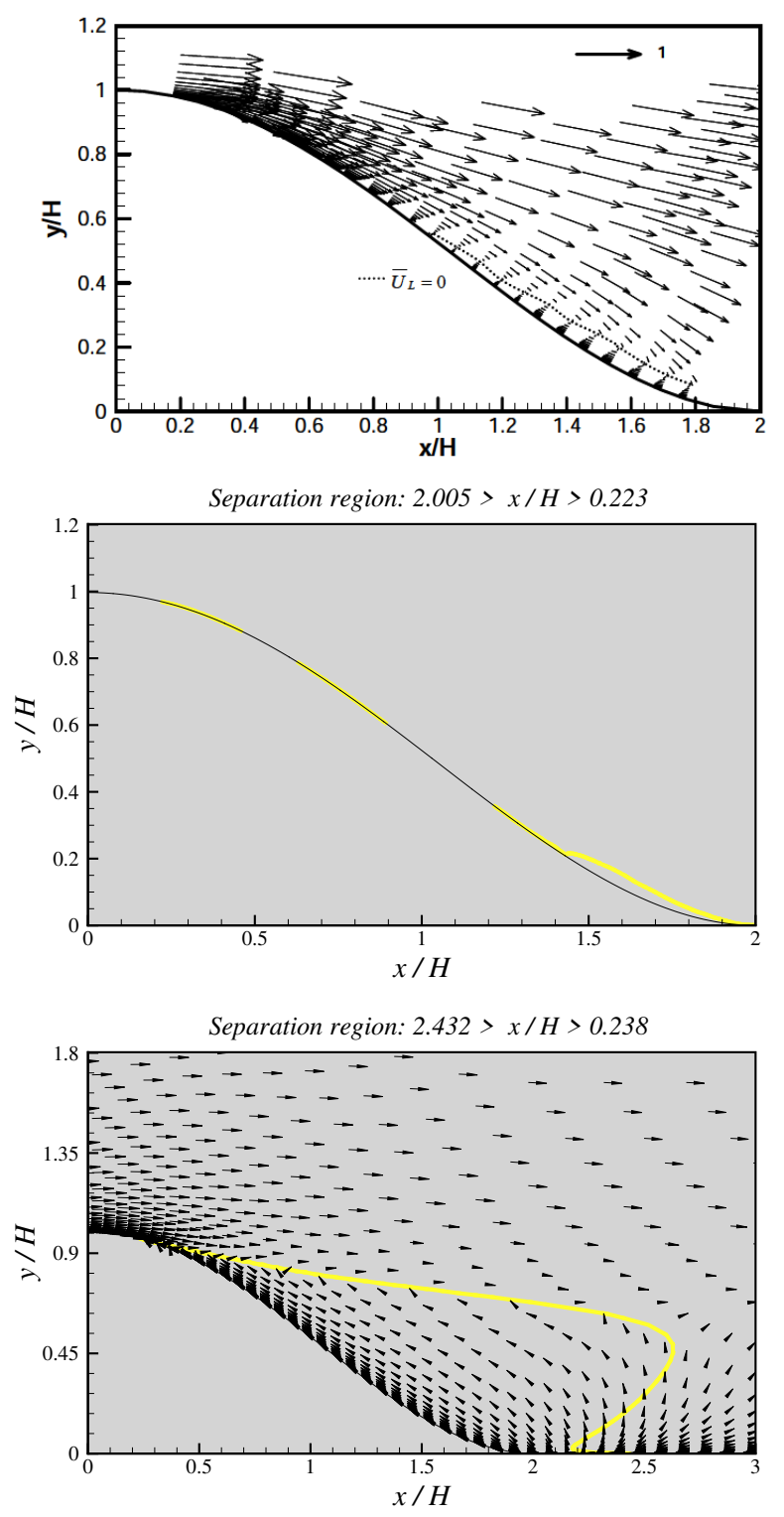

Fig. 16. Contour of the mean streamwise velocity $\langle u\rangle=0$ in the midspan $(z=0)$ plane as in experiment [6] (top frame) and from current LES (middle frame) and URANS (bottom frame). Inplane velocity vectors are shown for URANS simulation in the bottom frame.

and also the boundary condition used in the top wall. Furthermore, it is sensitive to the numerical accuracy of the flow solver.

The lee-side separation and reattachment zone in the midspan $(z=0)$ plane for the URANS simulation is shown in the bottom frame of Fig. 16. The recirculation region is depicted by plotting the $\langle u\rangle=0$ contour. The inplane mean velocity vectors have 
also been included for the URANS result. The recirculation region is in accordance with the URANS simulation results in $[10,19]$. URANS predicts the recirculation region to be much larger than the experiment. The separation and reattachment are incorrectly predicted by the URANS. Single-point URANS models are unsuccessful in predicting unsteady separational flows. This is because, the mean flow distortion length/ time scales are larger than the largest turbulent scales modeled by these models.
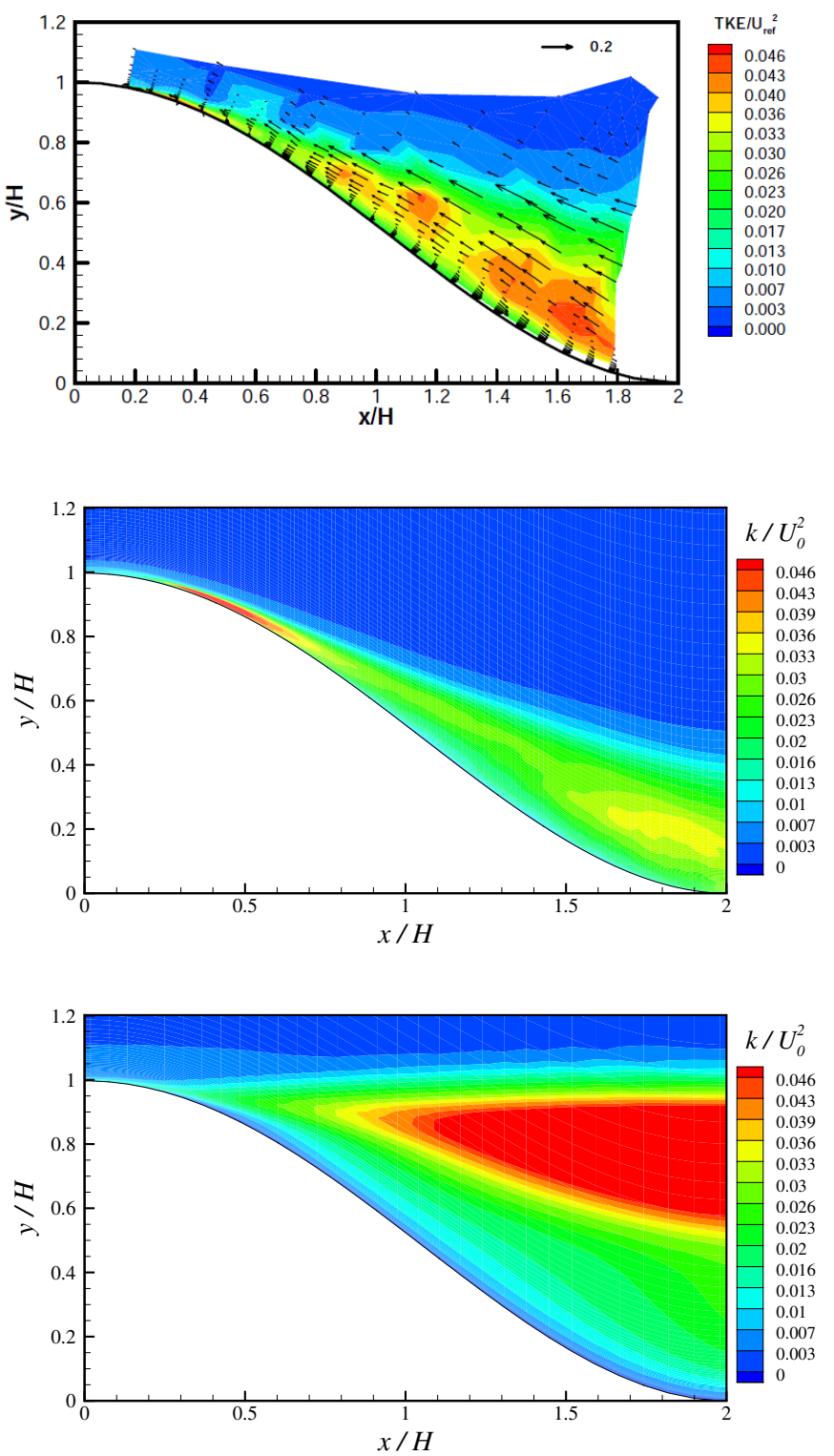

Fig. 17. Contours of the turbulent kinetic energy scaled by the reference velocity $\left(k / U_{0}^{2}\right)$ plotted in the midspan $(z=0)$ plane as in experiment [6] (top frame), from current LES (middle frame) and from the URANS simulation (bottom frame). 
Contours of $T K E$ have been plotted in the midspan $(z=0)$ plane from the experiment [7] and current LES in the top and middle frames of Fig. 17. A thin region of high turbulence activity is obtained very close to the wall from $x / H \sim 0.25$ onward in both the experiment and the current LES. In the bottom frame, this patch extends up to $x / H \sim 0.6$. In our simulation, separation is initiated at $x / H \sim 0.22$. So, in the LES, this patch is obtained just downstream of separation. It is understandable that after separation, the high velocity gradient due to the separated shear layer can significantly increase the turbulence production. Similar observation of a highly turbulent near-wall region after the initiation of separation in their high-resolution simulations was also reported in [14]. However, in the experiment, separation location is reported to be at $x / H \sim 0.96$. Therefore, LES is able to confirm that the separation initiation is indeed farther upstream compared with the separation location reported in the experiments. Right after separation, the recirculation region is very thin, which could not be captured in the experiment. This highly turbulent region belongs in the separated shear layer. Locally high turbulent contour values in the experiment depict the separated shear layer moving down the hill. Although, downstream of $x / H \sim 0.8$, values of local peak of $T K E$ are lower in the current LES, the wall-normal locations of the high contour values are in accordance with the experiment (this is not clearly visible from the contour plot in the bottom frame because of using same contour levels as the experiment). Therefore, it may be inferred that downstream evolution of the separated shear layer has been correctly captured by the LES. It was evident from the flow topology map in Fig. 14 that the recirculation region right after separation is very thin, and therefore, very difficult to capture correctly. Figure 17 demonstrates that the LES is able to capture the separated shear layer right above the recirculation region correctly.

For the URANS simulation, contours of $T K E\left(k / U_{0}^{2}\right)$ have been plotted in the symmetry plane in the bottom frame of Fig. 17. Although the range of $T K E$ seems to be correctly predicted, when compared with the experiment (see top frame of Fig. 17), $T K E$ is incorrectly predicted by the URANS. High $T K E$ is concentrated in the separated shear layer region depicted in Fig. 16.

The discussion above is mainly about the flow evolution, the separation and the recirculation specifically along the midspan plane in the lee-side of the hill. How does the boundary layer flow evolve in the cross-stream planes, i.e., along the spanwise extent of the hill? To help us understand the dynamics of the vortical structures and the consequence of the upstream topological features, one can plot the mean streamwise vorticity $\left\langle\omega_{x}\right\rangle=\frac{\partial\langle w\rangle}{\partial y}-\frac{\partial\langle v\rangle}{\partial z}$. This is done at various cross-stream $(y, z)$-planes at chosen streamwise stations downstream of the crest of the hill in Fig. 18 for the main LES. The signs of the vorticity are such that the streamwise flow is in the direction of the reader. The chosen streamwise stations belong in the range $0 \leq x / H \leq 2.5$, i.e., from upstream of separation to reattachment and also in the recovery region. The change of sign of $\left\langle\omega_{x}\right\rangle$ across $z=0$ in Figs. $18(a)$ and $18(b)$ indicate that $\left\langle\omega_{x}\right\rangle$ is anti-symmetric w.r.t. the midspan plane. That is why, to show a zoomed-in view of the boundary layer, only the left half of the spanwise extent of the hill is shown in Figs. 18(c) $-18(f)$.

Dominant boundary-layer vorticity upstream of the hill is oriented in the spanwise di- 

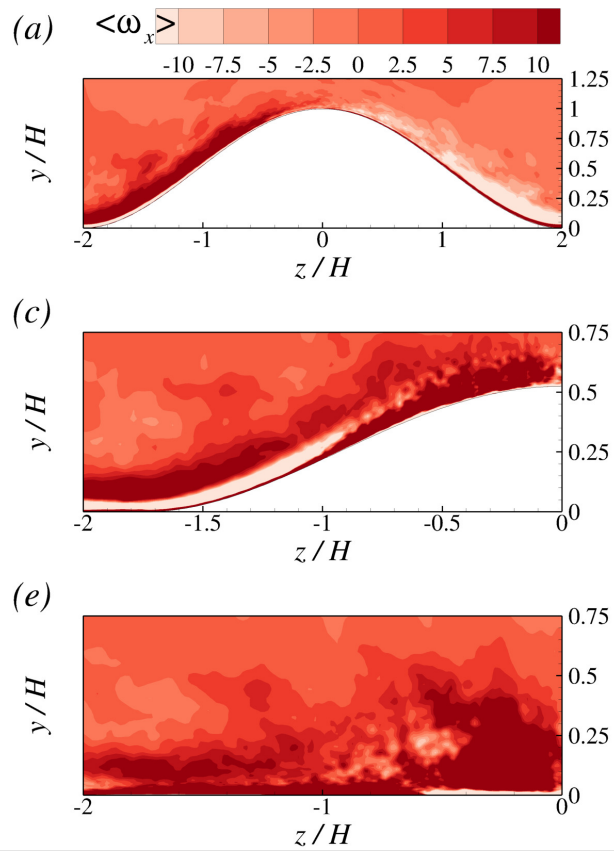

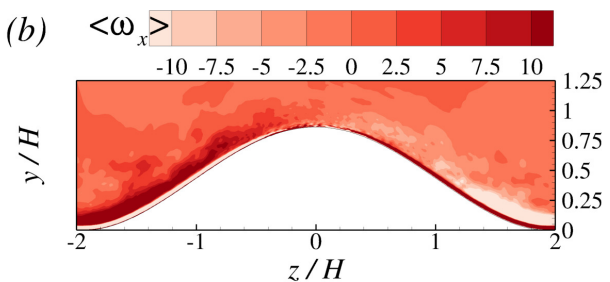

(d)
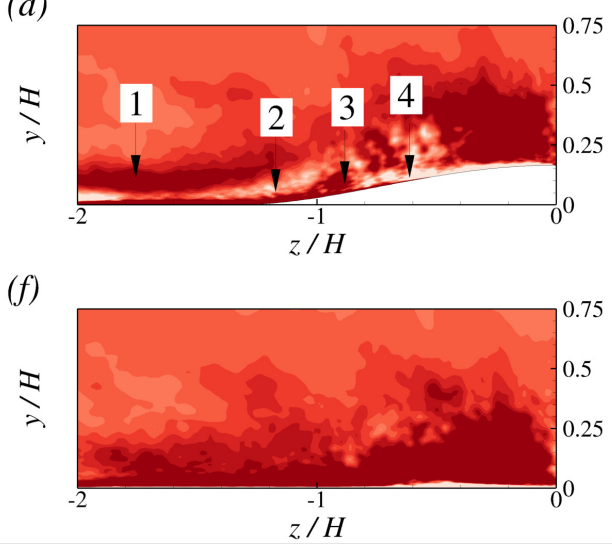

Fig. 18. Contours of mean streamwise vorticity $\left(<\omega_{x}>\right.$ in $\left.H z\right)$ plotted in wall-normal $(y, z)$-planes at chosen streamwise locations from the main LES. From $(a)$ to $(f)$, the streamwise locations are $x / H=0,0.5,1,1.5,2$ and 2.5 , respectively. Vorticity patches based on their origin upstream of the location $x / H=1.5$ are marked in frame $(d)$. See the main text for detail.

rection. Following flow acceleration in the upstream side, vorticity is reoriented in the streamwise direction. Consequently at the crest of the hill, in Fig. 18(a), dominant vorticity is positive (negative) in the left (right) half of the plane. This patch is marked as 1 in Fig. 18(d) which shows the contours at $x / H=1.5$. Beneath this strong vortical structures a very thin patch of vorticity with the opposite sign is visible very close to the wall. This layer gets even thinner as one moves towards the hill crest. This is due to the lower streamwise flow acceleration experienced by fluid away from the midspan location; the spanwise pressure gradient establishes weaker spanwise flow along that direction close to the wall. Similar characteristics of $\left\langle\omega_{x}\right\rangle$ are persistent downstream of the separation node at $x / H=0.5$ in Fig. 18(b). After the experimentally noted separation location, at $x / H=1$ in Fig. 18(c), the very thin layer of negative vorticity adjacent to the wall is displaced by a strong patch of positive $\left\langle\omega_{x}\right\rangle$ close to the midspan plane. This layer appears because of the separated shear layer from the separation node (see Fig. 14). This is marked as patch 3 in Fig. 18(d). This layer of vorticity becomes even stronger further downstream at $x / H=1.5$ as the recirculation region thickens which is marked as patch 4 in Fig. $18(d)$. In Fig. 18(d), patch 2 is the vorticity shed from the focus close to the wall. Further downstream, this vortical structure is dominated by the vortices from the separated shear layer (patch 3 vortices). At the reattachment location at $x / H=2$ in Fig. 18(e), the boundary- 
layer vorticity is mainly positive dominated by the upstream boundary-layer vorticity and the separated shear layer vorticity. Same is the case in the recovery region at $x / H=2.5$ in Fig. 18(f).
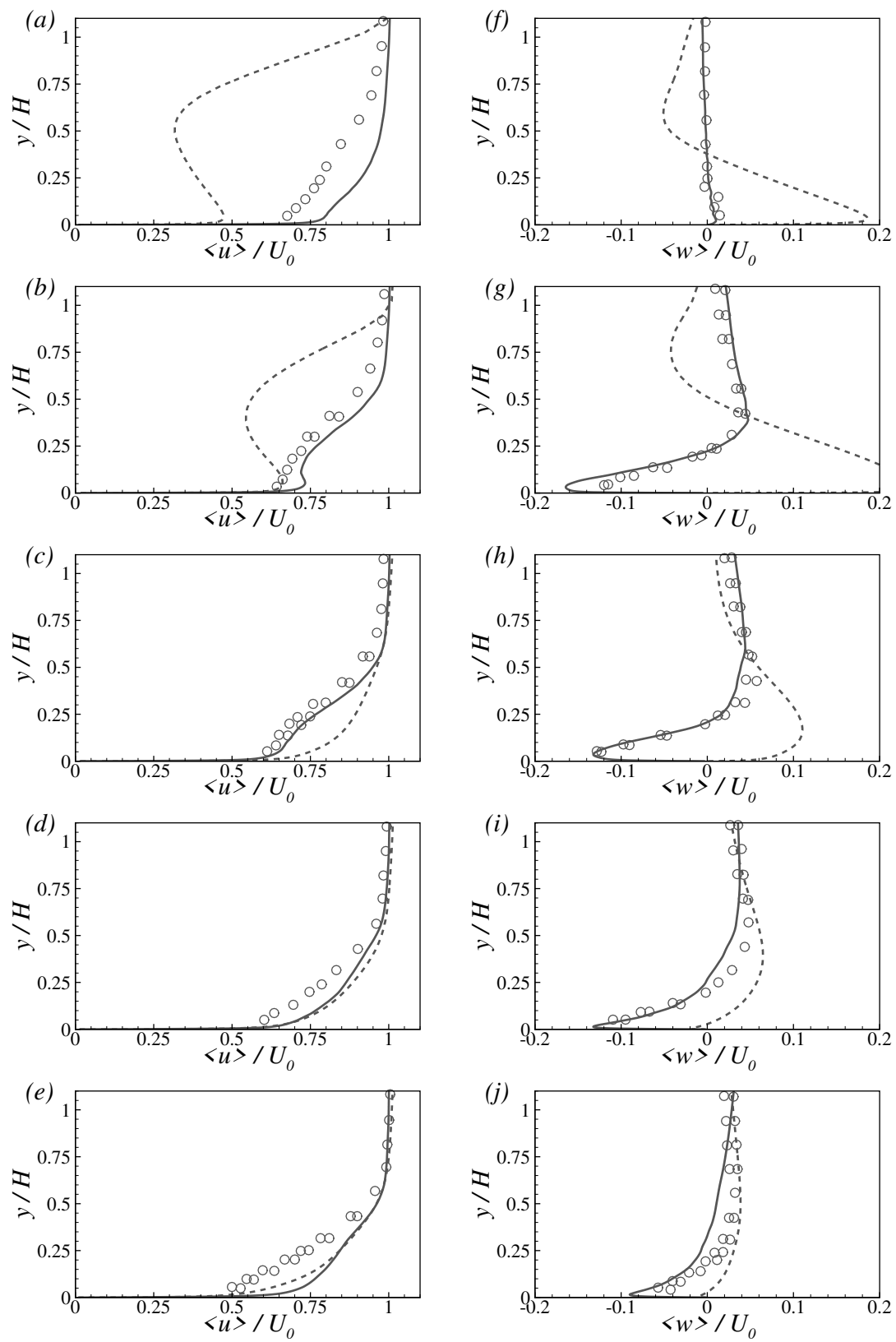

Fig. 19. Wallnormal profiles of the scaled time-mean $(a)-(e)$ streamwise $\left(<u>/ U_{0}\right)$ and $(f)-(j)$ spanwise $\left(\langle w\rangle / U_{0}\right)$ velocities at $x / H=3.63$ from LES (solid lines) and URANS (dashed lines) compared with experiment (symbols). From top to bottom the spanwise locations are $z / H=0,0.32,0.65,1.1,1.79$. 
The recovery of the flow is studied by plotting the wall-normal profiles of mean streamwise $(\langle u\rangle)$ and spanwise $(\langle w\rangle)$ velocities at several spanwise locations at $x / H=3.63$ in Fig. 19 and of TKE and cross Reynolds stress term $<u^{\prime} v^{\prime}>$ in Fig. 20. This streamwise station is farther downstream of the reattachment node in Fig. 14. These figures include the profiles from the LES (solid lines), URANS (dashed lines) and experiment (symbols). Overall, good quantitative agreement with the experiment is obtained. Due to the presence of the hill, streamwise momentum is reduced in the lee-side of the wake which is reflected in the inflectional profiles of $\langle u\rangle$ at $z / H=0.32$ and 0.65 . Profiles of $\langle w\rangle$ are also inflectional at spanwise stations away from the midspan location. Close to the wall, mean fluid motion is towards the symmetry plane, and farther away from the wall, fluid moves away from the symmetry plane as the profiles of $\langle w\rangle$ change sign in the wall-normal direction. This results in the inflectional profiles of $\langle u\rangle$. At the outermost spanwise station, significant overshoot is obtained for the current LES. This is probably due to coarse grid resolution in both streamwise and spanwise directions downstream of the hill.

The $\langle u\rangle$ profile at $z=0$ shows a large deficit in streamwise momentum in the current URANS in Figs. 19(a) and 19(b) contrary to the experiment/ LES in which the momentum deficit has already recovered. In the experiment, $\langle w\rangle$ profile is inflectional and changes sign in the wall-normal direction at all spanwise stations shown here. But for the URANS, change of sign is only obtained at $z / H=0$ and 0.32 for the $\langle w\rangle$ profile. Clearly, spanwise momentum transport is erroneous in URANS as it predicts the $\langle w\rangle$ component to change sign from negative to positive in URANS as one moves from free stream towards wall at $z / H=0.32$ which is exactly opposite to the experiment/ LES. A similar trend is also seen at $z / H=0$, where $\langle w\rangle$ significantly overshoots the experiment. The predictions are a little better farther away from $z / H=0$. Therefore, in separated, reattached and recovery regions, URANS predictions are unreliable.

As may be expected, the second order statistics presented from the current LES in Fig. 20 are in lesser agreement with the experimental profiles, specifically at spanwise stations close to the symmetry plane. Both $k$ and $\left\langle u^{\prime} v^{\prime}\right\rangle$ are underpredicted by the LES at $x / H=0$ and 0.32 . It is interesting to note that higher values of $T K E$ are obtained at $x / H=$ 0.32 instead of at $z / H=0$. However, at outer spanwise locations, i.e., at $x / H=0.65,1.1$ and 1.79, the profiles from current LES are in very good agreement with the experiment. Close to the symmetry plane, both $k$ and $\left\langle u^{\prime} v^{\prime}\right\rangle$ are inaccurately predicted by the URANS. As one moves further away from the symmetry plane, however, URANS predictions are in reasonable agreement with the experiment at $z / H=1.1$ and 1.79. Locations of peak values of $k$ are predicted well above the experimental peaks at $z / H=0$ and 0.32 by the URANS. URANS predicted signs for the $\left\langle u^{\prime} v^{\prime}\right\rangle$ component is opposite to the experiment at these locations. Evidently, LES predictions are much more reliable for this flow in the separational, post-reattachment recovery regions.

Frequency spectra have been plotted against the nondimensional frequency $f H / U_{0}$ in Figs. 21(a) and 21(b) for the $u$ and $w$ fluctuations, respectively, in the symmetry plane at the same streamwise station as in Figs. 19 and 20. The spectra from both the LES and experiment (symbols) are shown. The wall-normal location is at $y / H \sim 0.36$. This is 

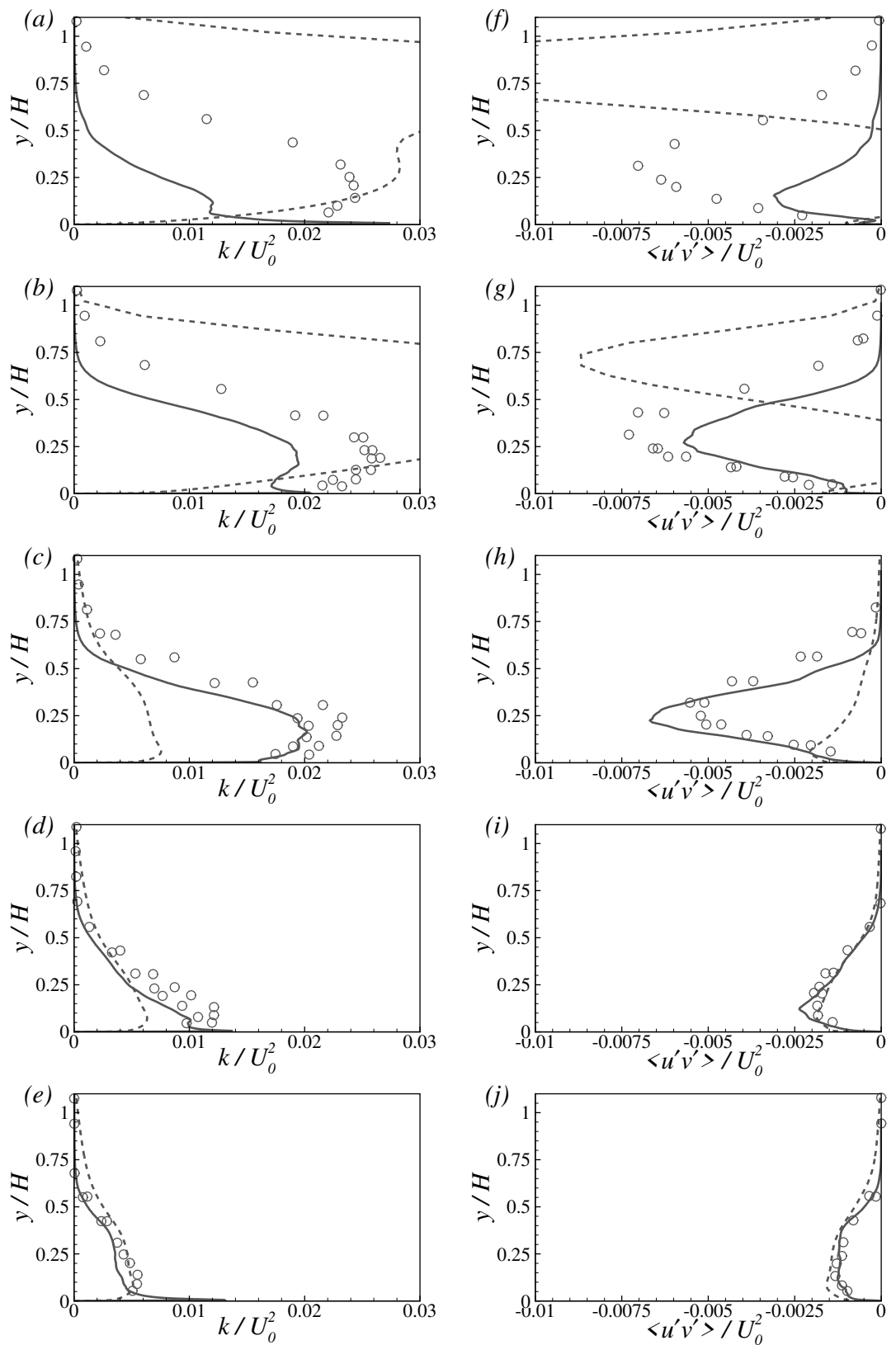

Fig. 20. Wallnormal profiles of the scaled $(a)-(e)$ turbulent kinetic energy $\left(k / U_{0}^{2}\right)$ and $(f)-(j)$ one of the cross components of the Reynolds shear stresses $\left(<u v>/ U_{0}^{2}\right)$ at $x / H=3.63$ from LES (solid lines) and URANS (dashed lines) compared with experiment (symbols). From top to bottom the spanwise locations are $z / H=0,0.32,0.65,1.1,1.79$.

around the region where the wall-normal peak in the $T K E$ profile is obtained in Fig.20. Data had been recorded for a time span of $264.42 \frac{H}{U_{0}}$. To obtain the spectra over a large range of frequency and at the same time to acquire some smoothness, the whole time span 

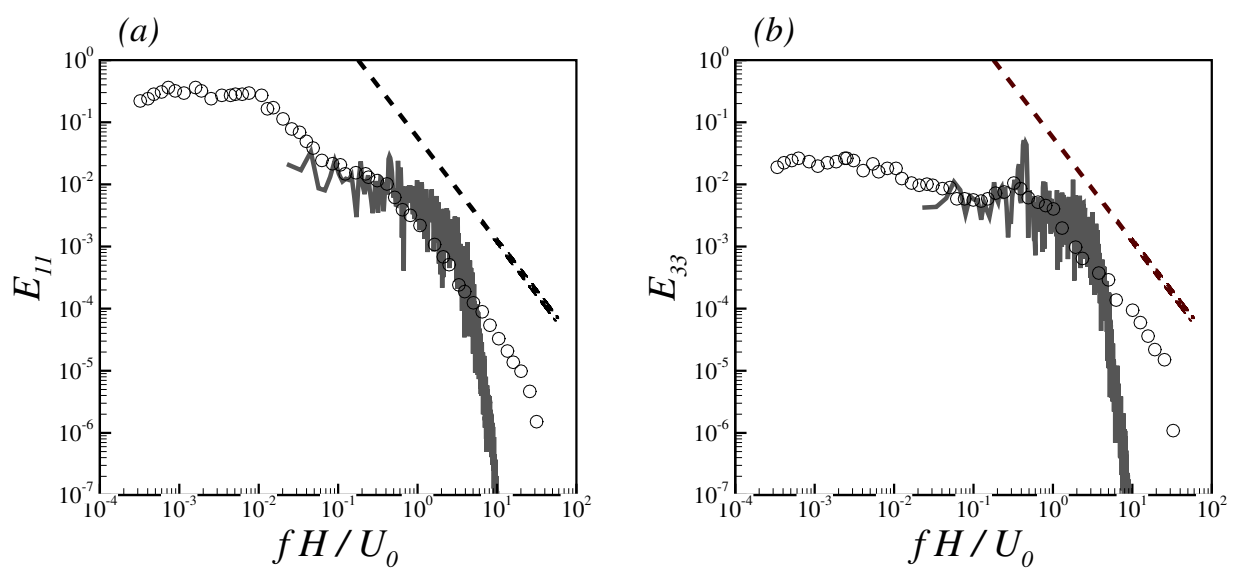

Fig. 21. Frequency spectra of the $(a)$ streamwise and $(b)$ spanwise velocity fluctuations at $x / H=3.63, y / H=0.358$ in the midspan $(z=0)$ plane compared with experiment (symbols). The dashed line corresponds to $-5 / 3-$ law.

of the recorded data has been split into three non-overlapping chunks of equal time span. The spectra from the experiment are still over a much larger range of frequency compared to the spectra obtained from the current LES. A Hann window was applied to each chunk of the data and then a Fast Fourier Transform (FFT) was applied to each chunk of data. The spectra shown are the mean of these three separately calculated FFTs. The spectra from LES drop off faster beyond $f H / U_{0} \sim 5$ because of the relatively large grid spacing compared to a standard well-resolved LES. Reasonable quantitative agreement with the experiment is obtained at lower frequencies $f H / U_{0}<0.1$. The slope of the spectra from LES is consistent with the isotropic range satisfying the $-5 / 3$ law over at least a decade and a half of the logarithmic frequency scale for the $u$ component and for at least a decade of the frequency range for the $w$ component. The spectra are over-predicted by the LES probably because of the numerical noise due to discretization error and resolved turbulence at the smallest grid unit. It is to be noted that a distinct peak is obtained at $f H / U_{0} \sim 0.42$, especially in the spectrum for the $w$ fluctuations in Fig. 21(b). This is probably due to the obtained peak in the spectra for the precursor simulation in Fig. 7 at $y+\sim 1500$ which was attributed to the imposed streamwise periodicity in a short streamwise domain used for the precursor simulation. Therefore, some effect of the time-periodicity of inlet turbulence is retained much further downstream emphasizing the importance of the inlet turbulence generation method.

\subsection{Upstream-side flow}

The URANS predictions are erroneous in the lee-side separation, recirculation and reattached flow undergoing recovery, especially close to the symmetry plane. How about URANS predictions in the upstream side of the hill? To gauge performance of URANS 

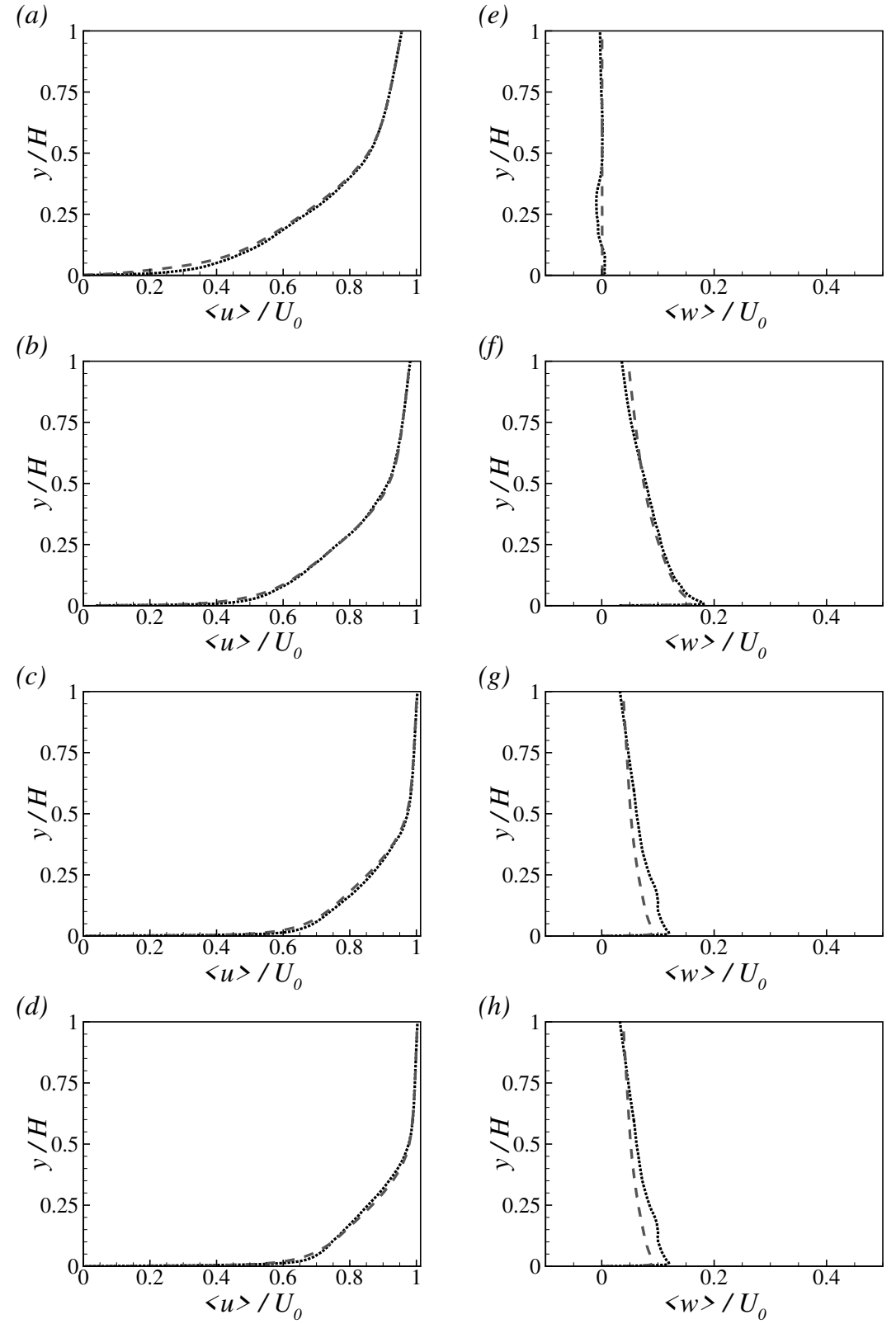

Fig. 22. Profiles of the scaled time-mean $(a)-(d)$ streamwise $\left(\langle u\rangle / U_{0}\right)$ and $(e)-(h)$ spanwise $\left.(<w\rangle / U_{0}\right)$ velocities at $x / H=-2$ in the $y$-direction from current URANS (dashed line) compared with the current LES (dotted line). From top to bottom the spanwise locations are $z / H=0,1,2,3$.

in the upstream side of the hill, mean streamwise and spanwise velocity profiles in the $y-$ direction have been plotted at three chosen streamwise stations in Figs. 22-24. The three chosen streamwise stations are $x / H=-2$ (right at the foot of the hill periphery), -1 (half 
way down the hill in the streamwise direction) and 0 (at hill crest). In each figure, profiles have been shown at spanwise stations $z / H=0,1,2$ and 3 in frames $(a)-(d)$ for the $<u>$-component, and in frames $(e)-(h)$ for the $<w>$-component, respectively. Close to the symmetry plane, URANS only very slightly under-predicts the $\langle u\rangle$ profiles in all of these plots. The LES profiles are fuller close to the wall indicating more turbulent activity. At the hill crest in Fig. 24(a), $<u>$ overshoots the free-stream velocity very close to the wall due to significant flow acceleration. Here too, w.r.t. the LES, URANS under-predicts the peak velocity by $\sim 8 \%$. Despite such discrepancies, overall, the URANS predictions are in good agreement with LES for the $\langle u\rangle$ profiles.

However, discrepancy in predictions is more pronounced for the $\langle w\rangle$ profiles. In the profiles shown in the cross-stream plane at the foot of the hill $(x / H=-2)$, expectedly, mean spanwise flow is outward, i.e., away from the hill symmetry plane. Again, the URANS under-predicts the $\langle w\rangle$ profiles compared to the LES. In the profiles shown in the $x / H=-1$ plane in Fig. 23, mean spanwise motion is also outward, which is maximum at $z / H=1$ because, the flow experiences the expanding extent of the hill at this streamwise location. This results in more fluid being ejected outward from close to the symmetry plane. Interestingly, the mean spanwise flow very close to the wall is towards the symmetry plane at $x / H=0$ as per the LES predictions. In contrast, in Figs. 24(e) and 24( $f)$, URANS predicts a mean outward flow. This is indeed evident from the streamlines shown from the skin-friction coefficients for LES and URANS predictions in Fig. 14. The mean streak-lines in those plots are towards the hill crest for the LES and away from the hill crest for the URANS. This indicates that the spanwise pressure gradient obtained in the URANS calculation is inaccurately leading to significant discrepancy. Farther away from the symmetry plane, in the flat region for example (at $z / H=2$ and 3), the predicted direction of mean spanwise motion is same for both LES and RANS. But the URANS predicts a lower peak velocity close to the wall at these stations.

In applied wind engineering of flow over complex topographies, the Topographic Speedup Factor $(T S F)$ at a chosen distance from the wall is defined as the ratio of the mean streamwise velocity at a chosen streamwise station on the hill to the mean streamwise velocity in absence of the hill. TSF at the crest of the hill has been plotted as a function of the wall-normal distance $(y / H)$ in Fig. 25. Close to the wall $T S F$ is large. At $y / H=0.0005$, LES predicts the $T S F \sim 5.85$. This large value is due to the flow acceleration very close to the wall in the upstream section of the hill. For the URANS simulation, at this wall-normal height $T S F \sim 4.75$. URANS underpredicts the $T S F$ at all wall-normal locations. For example, at $y / H=0.002(y / H=0.02), T S F$ predicted by URANS and LES are $\sim 2.55(2.18)$ and $\sim 3.67$ (2.41), respectively. Farther away from the wall, for $y / H>0.5$, the difference between predictions is small. 

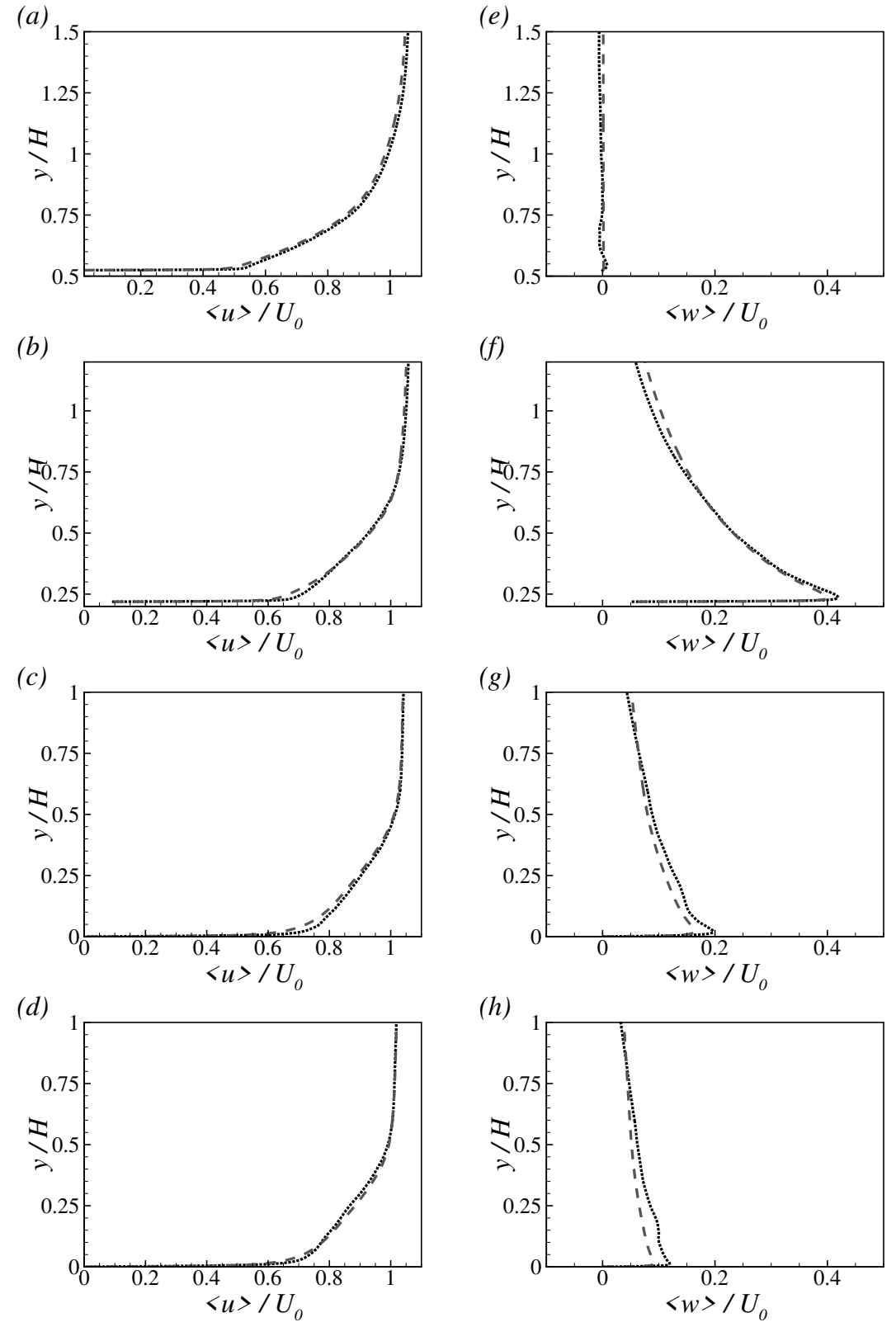

Fig. 23. Profiles of the scaled time-mean $(a)-(d)$ streamwise $\left(<u>/ U_{0}\right)$ and $(e)-(h)$ spanwise $\left.(<w\rangle / U_{0}\right)$ velocities at $x / H=-1$ in the $y$-direction from current URANS (dashed line) compared with the current LES (dotted line). From top to bottom the spanwise locations are $z / H=0,1,2,3$.

\section{Conclusion}

A couple of LES and an URANS simulations have been performed to study the separational flow behind a smooth-wall axisymmetric hill and the results are extensively compared with 

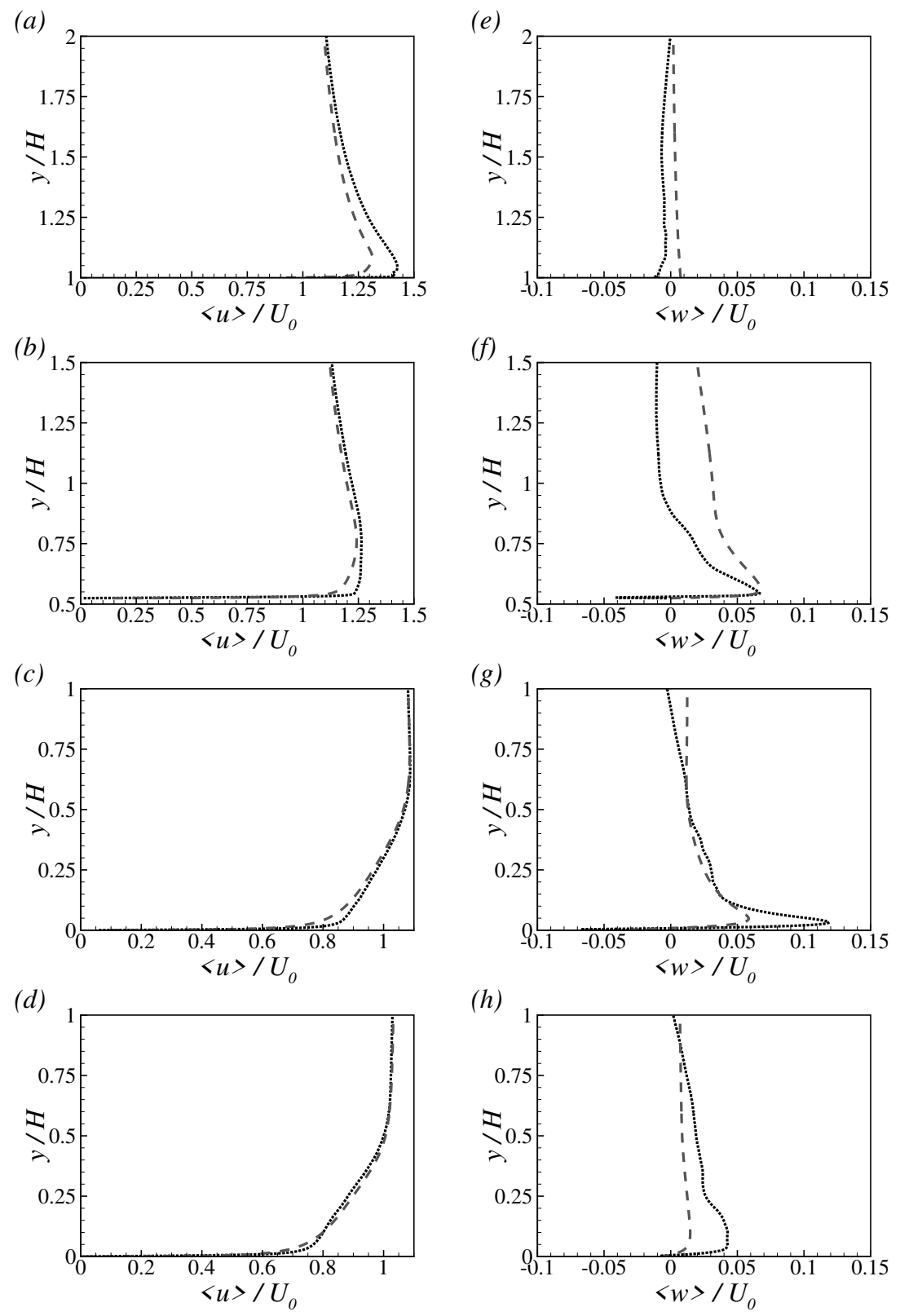

Fig. 24. Profiles of the scaled time-mean $(a)-(d)$ streamwise $\left(\langle u\rangle / U_{0}\right)$ and $(e)-(h)$ spanwise $\left(<w>/ U_{0}\right)$ velocities at $x / H=0$ in the $y$-direction from current URANS (dashed line) compared with the current LES (dotted line). From top to bottom the spanwise locations are $z / H=0,1,2,3$.

experiments of the same flow reported in [4, 7]. The Reynolds number w.r.t. hill height is $\operatorname{Re}_{H}=130,000$. For the LES, the dynamic Smagorinsky model [17] was used to model the SGS turbulent stresses which is widely used for separational flow calculations. To match the experimental conditions at the inflow of the main LES simulation domain with the 


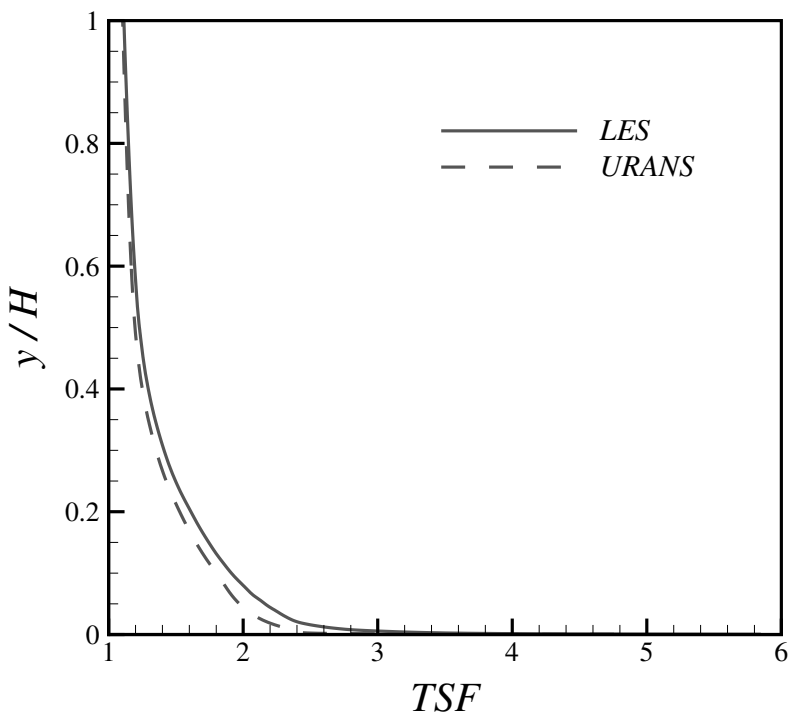

Fig. 25. Topographic Speed-up Factor (TSF) at the crest of the hill plotted as a function of wall-normal distance $(y / H)$ from current URANS (dashed line) compared with the current LES (solid line).

hill geometry, a separate precursor LES was performed. In this precursor LES, the mean velocity profile reported in the experiments was forced by including a forcing term on the RHS of the Navier-Stokes equations that varies in time and in the cross-stream planes. In the process, the precursor forcing techniques used to match experimental mean velocity and turbulent kinetic energy profiles [18] and the dynamic Smagorinsky SGS model have been implemented for the OpenFOAM wind engineering software, windOF. The spectra computed for the turbulent flow obtained from the precursor LES shows an extended region satisfying the isotropic $-5 / 3$-law at small scales.

The efficacy of the main LES was measured by calculating a total eddy viscosity based on the Reynolds stresses computed from the LES. Comparison of the mean SGS eddy viscosity with the total eddy viscosity shown in Fig. 10 demonstrates that the maximum SGS eddy-viscosity is only $\sim 1.5 \%$ of the total eddy viscosity indicating the contribution of the SGS eddy viscosity towards the computed Reynolds stresses is negligible which implies that the LES is accurate. In the process of validating the results with the experimental results, several flow parameters have been studied. $C_{p}$ distribution showed very good agreement with the experiment and other high-resolution LES (see Figs. 12 and 13). The flow topology was investigated by plotting the mean streak-lines at various wall-parallel planes in Figs. 14 and 15. These figures depict the mean separation dynamics in great detail. In total five nodes and five saddle points were obtained from our LES which is in contradiction with the flow topology reported in [14] who reported presence of four nodes and 
saddle points. The discrepancy is reflected in a thinner recirculation region predicted in the midspan plane as shown in Fig. 16. The separated region is highly intermittent and sensitive to almost all the flow attributes, such as, the incoming turbulence, boundary condition imposed at the top wall, grid resolution and stretching, etc. Such sensitivity makes it extremely difficult to predict the separated flow in the lee-side of the hill correctly. Further investigations were carried out for the recovering flow by plotting the mean streamwise vorticity at several lee-side cross-stream planes in Fig. 18. It was found that the vorticity in this region is dominated by the vorticity from the separated shear layer and the boundary-layer vorticity upstream of separation. Wallnormal profiles of first and second order statistics plotted in Figs. 20 and 21 from the main LES showed very good agreement with experimental results.

Widely used $k-\omega$ SST turbulence model was used for the URANS simulation. The results are in good agreement with another URANS calculation with the same model of the same flow by other researchers [19]. However, significant discrepancies, both qualtitative and quantitative are obtained in the separation region. The flow topology predicted by the URANS in the bottom frame of Fig. 14 is very different from the one obtained from LES. Furthermore, the predicted recirculation zone is very thick and unphysical (see the bottom frame of Fig. 16). Flow statistics presented for the URANS calculation in the recovery region also shows significant quantitative discrepancies with the experiment. For example, URANS predictions for the direction of the mean spanwise flow is erroneous in the region right behind the hill in Figs. $19(f)-19(h)$. Farther away from the midspan plane, URANS predictions improve. The mean streamwise and spanwise flow profiles obtained from URANS and LES were compared in Figs. 22-24 at three cross-stream planes in the upstream portion of the hill where the flow accelerates. URANS slightly underpredicts the mean streamwise profiles in this region. However, the most notable discrepancy was the mean spanwise flow towards the midspan plane very close to the wall predicted by the LES at $x / H=0$ in Fig. 24, while a mean outward spanwise flow away from the hill was predicted by URANS. Finally, Topographic Speed-up Factor (TSF) plotted against wallnormal distance $(y / H)$ in Fig. 25 at the crest of the hill indicates large flow acceleration close to the wall w.r.t. the flow in absence of the hill. URANS underpredicts $T S F$ compared to the LES at all wall-normal stations. Farther away from the wall, for $y / H>0.5, T S F$ predicted by LES and URANS show very little difference.

Present study of a thin boundary layer flow over an axisymmetric hill (boundary-layer thickness is only half of the hill height; geophysical equivalence would be a flow over a high mountain) has two implications from an engineering point of view. Firstly, URANS predicts the upstream-side flow reasonably well providing quicker solution using much less computational resources compared to an LES. Although URANS underpredicts the mean quantities (e.g., TSF predicted by URANS is at least $20 \%$ lower close to the wall), it may be useful for a qualitative measure of the approach flow. Secondly, for an accurate prediction of flow characteristics in the lee-side of the hill, well-resolved LES is necessary. The separation dynamics in this complex flow over a 3-D continuous wall is extremely sensitive to the CFD method resolving the flow dynamics accurately; which in turn, affects 
the accuracy of the solution in the separation, reattachment and recovery regions of the flow.

\section{Acknowledgments}

The authors wish to thank Dr. Emil Simiu of the National Institute of Standards and Technology for his insightful discussions and helpful comments on the draft of this report. Dr. Rikhi Bose served as a NIST Director's Postdoctoral Researcher. 


\section{References}

[1] Byun G, Simpson RL (2006) Structure of three-dimensional separated flow on an axisymmetric bump. AIAA J 44(5):999-1008.

[2] Byun G (2005) Sturcture of Three-Dimensional Separated Flow on Symmetric Bumps. Ph.D. thesis. Virginia Tech, .

[3] Garcia-Villalba M, Li N, Rodi W, Leschziner M (2009) Large-eddy simulation of separated flow over a three-dimensional axisymmetric hill. J Fluid Mech 627:55-96.

[4] Jamal T, Walters DK (2016) Simulation of a 3d axisymmetric hill: Comparison of rans and hybrid rans-les models. ASME 2016 Fluids Engineering Division Summer Meeting collocated with the ASME 2016 Heat Transfer Summer Conference and the ASME 2016 14th International Conference on Nanochannels, Microchannels, and Minichannels (American Society of Mechanical Engineers), , pp V01BT25A010V01BT25A010.

[5] Weller HG, Tabor G, Jasak H, Fureby C (1998) A tensorial approach to computational continuum mechanics using object-oriented techniques. Comput Phys 12(6):620-631.

[6] Shi L, Shi L, Yeo D (2016) OpenFOAM large-eddy simulations of atmospheric boundary layer turbulence for wind engineering applications (US Department of Commerce, National Institute of Standards and Technology), .

[7] Yeo D, Shi L (2018) Computational versus wind tunnel simulation of atmospheric boundary layer flow for structural engineering applications. Wind Engineering for Natural Hazards: Modeling, Simulation, and Mitigation of Windstorm Impact on Critical Infrastructure, , pp 169-191.

[8] Simpson RL, Long CH (2001) Study of vortical separation from an axisymmetric hill. TSFP DIGITAL LIBRARY ONLINE (Begel House Inc.), , .

[9] Ma R, Simpson RL (2005) Characterization of turbulent flow downstream of a threedimensional axisymmetric bump. Tsfp Digital Library Online (Begel House Inc.), ,

[10] Wang C, Jang Y, Leschziner M (2004) Modelling two-and three-dimensional separation from curved surfaces with anisotropy-resolving turbulence closures. Int J Heat Fluid Fl 25(3):499-512.

[11] Davidson L, Dahlström S (2005) Hybrid les-rans: Computation of the flow around a three-dimensional hill. Engineering Turbulence Modelling and Experiments 6 (Elsevier), , pp 319-328.

[12] Persson T, Liefvendahl M, Bensow R, Fureby C (2006) Numerical investigation of the flow over an axisymmetric hill using les, des, and rans. J Turbul (7):N4.

[13] Patel N, Menon S (2007) Structure of flow separation and reattachment behind an axisymmetric hill. $J$ Turbul (8):N36.

[14] Tessicini F, Li N, Leschziner M (2007) Large-eddy simulation of three-dimensional flow around a hill-shaped obstruction with a zonal near-wall approximation. Int J Heat Fluid Fl 28(5):894-908.

[15] Krajnović S (2008) Large eddy simulation of the flow over a three-dimensional hill. 
Flow, Turbulence and Combustion 81(1-2):189-204.

[16] García-Villalba M, Wissink JG, Rodi W (2010) Influence of the approach boundary layer on the flow over an axisymmetric hill at a moderate reynolds number. $J$ Turbul (11):N8.

[17] Germano M, Piomelli U, Moin P, Cabot WH (1991) A dynamic subgrid-scale eddy viscosity model. Physics of Fluids A: Fluid Dynamics 3(7):1760-1765.

[18] Lilly DK (1992) A proposed modification of the germano subgrid-scale closure method. Physics of Fluids A: Fluid Dynamics 4(3):633-635.

[19] Pierce CD, Moin P (2001) Progress-variable approach for large-eddy simulation of turbulent combustion. Ph.D. thesis. Citeseer, .

[20] Menter FR, Kuntz M, Langtry R (2003) Ten years of industrial experience with the sst turbulence model. Turbulence, heat and mass transfer 4(1):625-632.

[21] Davidson L (2009) Large eddy simulations: how to evaluate resolution. Int J Heat Fluid Fl 30(5):1016-1025.

[22] Choi H, Moin P (1994) Effects of the computational time step on numerical solutions of turbulent flow. Journal of Computational Physics 113(1):1-4.

[23] Courant R, Friedrichs K, Lewy H (1928) Über die partiellen differenzengleichungen der mathematischen physik. Mathematische annalen 100(1):32-74.

[24] Counihan J (1975) Adiabatic atmospheric boundary layers: a review and analysis of data from the period 1880-1972. Atmospheric Environment (1967) 9(10):871-905.

[25] Durbin PA, Reif BP (2011) Statistical theory and modeling for turbulent flows (John Wiley \& Sons), .

[26] Hunt J, Abell C, Peterka J, Woo H (1978) Kinematical studies of the flows around free or surface-mounted obstacles; applying topology to flow visualization. J Fluid Mech 86(1):179-200. 